\title{
The Infectious Disease Ontology in the Age of COVID-19
}

\author{
Shane Babcock ${ }^{1,5^{*}}$, John Beverley ${ }^{2,5}$, Lindsay G. Cowell ${ }^{3,5}$, Barry Smith ${ }^{4,5}$ \\ ${ }^{1}$ Department of Philosophy, Niagara University, Lewiston, NY, USA \\ ${ }^{2}$ Department of Philosophy, Northwestern University, Evanston, IL, USA \\ ${ }^{3}$ Cowell Lab, University of Texas Southwestern Medical Center, Dallas, TX, USA \\ ${ }^{4}$ Department of Philosophy, University at Buffalo, Buffalo, NY, USA \\ ${ }^{5}$ National Center for Ontological Research, University at Buffalo, Buffalo, NY, US \\ * Correspondence: \\ Shane Babcock \\ sbabcock@niagara.edu
}

This a preprint version of an article to appear in Journal of Biomedical Semantics.

\begin{abstract}
Background: Effective response to public health emergencies, such as we are now experiencing with COVID-19, requires data sharing across multiple disciplines and data systems. Ontologies offer a powerful data sharing tool, and this holds especially for those ontologies built on the design principles of the Open Biomedical Ontologies Foundry. These principles are exemplified by the Infectious Disease Ontology (IDO), a suite of interoperable ontology modules aiming to provide coverage of all aspects of the infectious disease domain. At its center is IDO Core, a disease- and pathogen-neutral ontology covering just those types of entities and relations that are relevant to infectious diseases generally. IDO Core is extended by disease and pathogen-specific ontology modules.
\end{abstract}

Results: To assist the integration and analysis of COVID-19 data, and viral infectious disease data more generally, we have recently developed three new IDO extensions: IDO Virus (VIDO); the Coronavirus Infectious Disease Ontology (CIDO); and an extension of CIDO focusing on COVID19 (IDO-COVID-19). Reflecting the fact that viruses lack cellular parts, we have introduced to IDO Core the term acellular structure to cover viruses and other acellular entities studied by virologists. We now distinguish between infectious agents - organisms with an infectious disposition - and infectious structures - acellular structures with an infectious disposition. This in turn has led to various updates and refinements of IDO Core's content. We believe that our work on VIDO, CIDO, and IDOCOVID-19 can serve as a model for yielding greater conformance with ontology building best practices.

Conclusions: IDO provides a simple recipe for building new pathogen-specific ontologies in a way that allows data about novel diseases to be easily compared, along multiple dimensions, with data represented by existing disease ontologies. The IDO strategy, moreover, supports ontology coordination, providing a powerful method of data integration and sharing that allows physicians, researchers, and public health organizations to respond rapidly and efficiently to current and future public health crises.

Keywords: coronavirus, COVID-19, infectious disease, Infectious Disease Ontology, ontology, data integration

\section{Background}


Efforts by physicians, researchers, and public health organizations to respond to infectious diseases require the use of multiple, constantly changing data sources. Consider, for instance, a research team trying to model a given population's herd immunity to measles. This depends on the integration of data not merely from biology and medicine, but also from public health, geography, and social science [1]. Because such data is collected using discipline- and community-specific methodologies and is stored in geographically distributed and often non-interoperable databases, the data are typically only locally accessible. The resultant silo-formation [2] hinders both translational and comparative research and preventive and prognostic public health research [3]. These problems can be solved by traditional means with the investment of sufficient time and effort. In circumstances of public health emergency, however, more powerful methods for data sharing and integration must be applied.

As the experience of biologists and bioinformaticians has shown, ontologies - logically welldesigned, structured, vocabularies - are a powerful data sharing tool [4]. But to be effective, ontologies must be designed in a coordinated fashion - otherwise ontologies themselves will give rise to the creation of a new kind of silo [2]. One of the most successful and widely adopted approaches to coordinated ontology development is that of the Open Biomedical Ontologies (OBO) Foundry [5], a collective of developer groups dedicated to creating, testing, and maintaining a suite of ontologies based on an evolving set of ontology design principles:

- Ontologies should use a well-specified syntax and share a common space of identifiers.

- Ontologies should be openly available in the public domain for reuse.

- Ontologies in neighboring domains should be developed in a collaborative effort.

- Ontologies should be developed in a modular fashion.

- Ontologies should have a clearly specified scope.

- Ontologies should use common unambiguously defined relations between their terms.

- Ontologies should conform to a common top-level architecture.

The OBO Foundry principles were modelled initially on the practices of the Gene Ontology (GO) [4], which has served as the model for subsequent life science ontologies [6].

Wherever possible, OBO ontologies are created using terms, relational expressions, and definitions taken from existing OBO ontologies, including the Relation Ontology (RO) [7], which ensures cross-linkage between ontologies in neighboring domains and also helps avert redundant efforts. Basic Formal Ontology (BFO) is the official top-level ontology for all OBO Foundry ontologies. BFO is comprised of highly general classes such as 'object', 'material entity', and 'process', is used by more than 350 ontology projects as their top-level architecture [2] and has been approved as international standard ISO/IEC 21838-2 [8, 9]. Ontology construction and extension in accordance with OBO principles follows a 'hub and spokes' model, where a core or 'hub' ontology provides the basis for extension ontologies providing domain-specific terms at progressively lower levels. The Infectious Disease Ontology (IDO), first released in 2010 [10], was constructed in this manner, and consequently, provides a central 'hub' from which 'spoke' ontologies extend to more specific disease domains.

\section{IDO Core, BFO and OGMS}

IDO Core covers just those entities that are relevant to infectious diseases generally, and not to specific infectious diseases associated with specific pathogens. Its coverage ranges across biological scales (gene, cell, organ, organism, population), disciplinary perspectives (biological, clinical, 
epidemiological), and successive stages along the chain of infection (host, reservoir, vector, pathogen) [11]. At the heart of IDO Core is the term 'disease', which is imported from the Ontology for General Medical Science (OGMS) [12]. Developers of OGMS view the traditional practice of classifying diseases according to patterns of similarities in signs and symptoms as inadequate. A single disease may manifest a variety of symptoms, making it difficult to distinguish the disease definitionally from other diseases involving the same anatomical system [13]. To address such issues, OGMS characterizes diseases in BFO terms as dispositions of patients to undergo pathological processes of specific kinds. Distinguishing manifestations of symptoms from dispositions to manifest symptoms provides the flexibility needed to represent diseases that have multiple different sorts of presentation [12]. The OGMS approach allows, moreover, for the existence of pre-clinical manifestations of disease, and for clinical risk factor combinations of disease and predispositions to disease (as when AIDS in a given patient is a risk factor for a second disease such as tuberculosis [14]). Supplementary Table 1 and Additional File 1 detail ontologies which make use of the OGMS approach to disease. Table 1 below reflects OGMS definitions relevant to our discussion of IDO Core.

Table 1| Definitions imported from OGMS to IDO Core

\begin{tabular}{||l||l||}
\hline \hline \multicolumn{1}{|c||}{ OGMS Term } & \multicolumn{1}{c|}{ Definition } \\
\hline \hline disease & $\begin{array}{l}\text { Disposition to undergo pathogenic processes that exists in an } \\
\text { organism because of one or more disorders in that organism. }\end{array}$ \\
\hline \hline disease course & $\begin{array}{l}\text { Totality of all processes through which a given disease instance } \\
\text { is realized. }\end{array}$ \\
\hline \hline disorder & $\begin{array}{l}\text { Material entity which is clinically abnormal and part of an } \\
\text { extended organism; disorders are the physical basis of disease. }\end{array}$ \\
\hline \hline symptom & $\begin{array}{l}\text { Process experienced by the patient, which can only be } \\
\text { experienced by the patient, that is hypothesized to be clinically } \\
\text { relevant. }\end{array}$ \\
\hline
\end{tabular}

The term disease relies on organism in its definition. The class organism is imported from the Ontology for Biomedical Investigations (OBI) [16] and defined as "an object that is an individual living system, such as animal, plant, bacteria, or virus, that is capable of replicating or reproducing, growth and maintenance in the right environment. An organism may be unicellular or made up, like humans, of many billions of cells divided into specialized tissues and organs." (As we shall see in $\mathbf{1 . 2}$ below, the reference to 'virus' here is problematic. We are working with the OBI developers to address this matter [17].) The term disorder relies on the class extended organism and elucidation of "clinically abnormal" in its definition. Instances of extended organism are object aggregates consisting of an organism and all material entities located within that organism overlapping the organism or occupying sites formed in part by the organism. Extended organism comprises not only the organism itself but also the normal microflora and invading pathogens contained within it and the pathogens on its surface, as well as the sites, for example the oral or nasal cavities, which these pathogens may occupy. Clinical abnormality is a feature of an organism that is not part of the life plan for an organism of the relevant type (unlike aging or pregnancy), is causally linked to an elevated risk either of pain or other feelings of illness, or of death or dysfunction, and is such that the elevated risk exceeds a certain threshold level [12].

\section{Results}


Recent development of three IDO extension ontologies - the Virus Infectious Disease Ontology, the Coronavirus Infectious Disease Ontology, and IDO-COVID-19 - has proceeded concurrently with updates and refinements to IDO Core's existing content, as well as new term imports from related OBO Ontologies. Section 1 details a selection of the relevant updates. Section 2 details the development of VIDO, CIDO and IDO-COVID-19.

\section{Extending IDO Core from OGMS}

The IDO Core extension of relevant classes from OGMS is represented visually in Figure 1 and with textual definitions in Table 2. Subclasses of entities are linked by BFO relations such as realizes and has_material_basis, the latter used to indicate the material basis of a disposition, in this case, a disease.

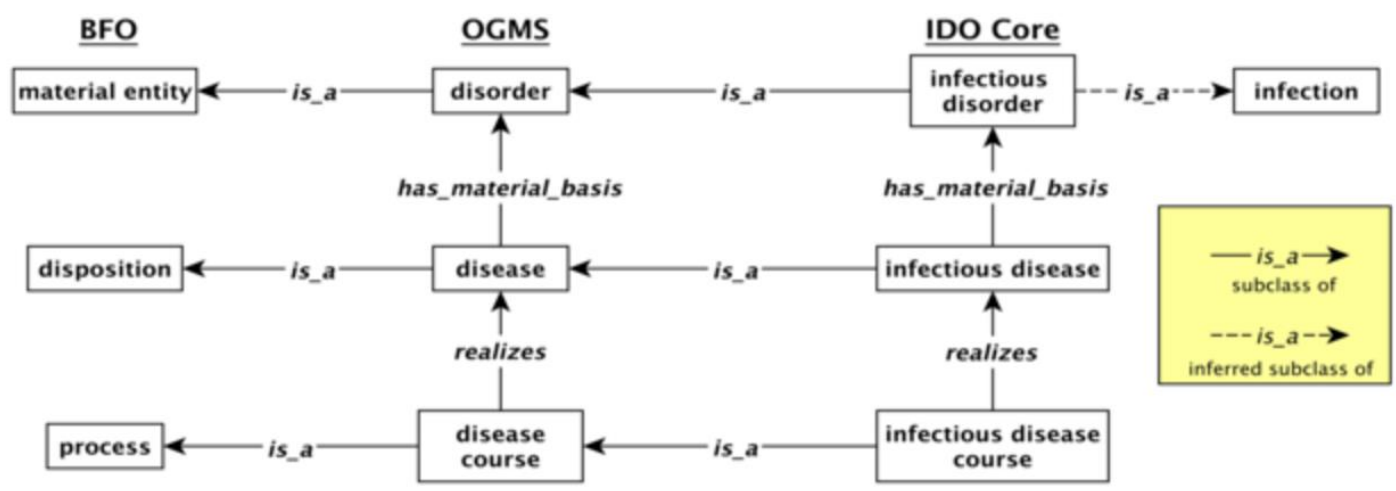

Figure 1 | Relationships between disease, disorder, and disease courses in IDO Core

Table 2| IDO Core definitions extending from OGMS

\begin{tabular}{||l||l||}
\hline \hline \multicolumn{1}{|c||}{ IDO Core Term } & \multicolumn{1}{c|}{ Definition } \\
\hline \hline infection & $\begin{array}{l}\text { Material entity part of an extended organism that has some } \\
\text { pathogen as part, which participates in the formation of the } \\
\text { material entity by invading tissues of the organism. }\end{array}$ \\
\hline \hline infectious disorder & $\begin{array}{l}\text { Disorder that is part of an extended organism which has an } \\
\text { infectious pathogen part, that exists as a result of a process of } \\
\text { formation of disorder initiated by the infectious pathogen. }\end{array}$ \\
\hline \hline infectious disease & Disease whose physical basis is an infectious disorder. \\
\hline \hline infectious disease course & Disease course that is the realization of an infectious disease. \\
\hline \hline
\end{tabular}

\subsection{Pathogens and Infectious Entities}

The term infection relies on pathogen, which is defined in IDO Core as a material entity bearing a pathogenic disposition. The term infectious disorder relies on infectious pathogen, which IDO Core defines as a pathogen bearing an infectious disposition. Corresponding updates in the most recent version of IDO Core are illustrated in Table 3.

Table 3 IDO Core definitions of infectious pathogens

\begin{tabular}{||c||c||}
\hline \hline \multicolumn{1}{|c||}{ IDO Core Term } & \multicolumn{1}{c|}{ Definition } \\
\hline \hline acellular structure & $\begin{array}{l}\text { Object consisting of an arrangement of interrelated } \\
\text { acellular parts forming an acellular biological unit that is } \\
\text { able to initiate replication of the structure in a host. }\end{array}$ \\
\hline
\end{tabular}




\begin{tabular}{||l|l||}
\hline \hline pathogenic disposition & $\begin{array}{l}\text { Disposition borne by a material entity to establish } \\
\text { localization in or produce toxins that can be transmitted to } \\
\text { an organism or acellular structure, either of which may } \\
\text { form disorder in the entity or immunocompetent members } \\
\text { of the entity's species. }\end{array}$ \\
\hline \hline infectious disposition & $\begin{array}{l}\text { Pathogenic disposition borne by a pathogen to be } \\
\text { transmitted to a host and then become part of an infection } \\
\text { in that host or immunocompetent members of the same } \\
\text { species as the host. }\end{array}$ \\
\hline \hline establishment of localization in host & $\begin{array}{l}\text { Process in which a material entity reaches a site in or on a } \\
\text { host in which it can survive, grow, multiply, or mature and } \\
\text { establishes itself there. }\end{array}$ \\
\hline \hline process of establishing an infection & $\begin{array}{l}\text { Process by which an infectious agent or infectious } \\
\text { structure, established in a host, becomes part of an } \\
\text { infection in the host. }\end{array}$ \\
\hline \hline appearance of disorder & Process through which a disorder comes into existence. \\
\hline
\end{tabular}

Motivation for these updates stems from reflection on the fact that importing the term organism from OBI implies viruses fall under this class and are cellular entities. Viruses are, however, acellular. To avoid this issue, IDO Core introduces the term acellular structure instances of which lack cellular parts, as a parent class for the class virus which is imported from NCBITaxon [18]. IDO Core now distinguishes between infectious agents - organisms bearing an infectious disposition - and infectious structures - acellular structures bearing an infectious disposition. The use of "organism or acellular structure" in pathogenic and infectious dispositions reflects, moreover, the fact that viruses themselves may be the targets of infection by, say, other viruses.

The definition of pathogenic disposition refers to the ability of a pathogen to participate in an establishment of localization in host, which can often form disorder if the subsequent maturing and/or multiplying of the pathogen in the host leads to tissue damage. The definition's first disjunctive clause covers cases where pathogens cause disorder without ever localizing in a host, as when foodborne toxins produced by clostridium botulinum are ingested, leading to food botulism. The definition also covers cases involving both mechanisms, as when the intestines of infants are colonized by $C$. botulinum and secreted toxins are then absorbed into the bloodstream.

The definition's second disjunctive clause allows for cases of localization that do not lead to disorders but are nevertheless contagious. An example would be the localization of HIV-1 in a human host that is resistant to the virus due to a mutation of the CCR-5 gene that blocks the virus from attaching to host cells, and so blocks pathogenesis to AIDS [19]. The virus's presence is not clinically abnormal as it is not causally linked to an elevated risk of either pain or other feelings of illness, or of death or dysfunction in the resistant host. But while the virus is unable to fully realize its infectious disposition in the host, it is still disposed to transmit to and bring clinical abnormality to other potential immunocompetent human hosts without the mutation. ${ }^{1}$

In making infectious disposition a child of pathogenic disposition, IDO Core distinguishes pathogenicity and infectiousness. C. botulinum, for example, is a pathogen, but not infectious. After an infant ingests honey colonized by $C$. botulinum, the bacterium secretes toxins into the bloodstream, resulting in disorder. But it does not itself become part of that disorder, nor is it disposed to be transmitted to new potential hosts. And so infant botulism is non-infectious. By contrast, COVID-19 is infectious

\footnotetext{
${ }^{1}$ Individuals with CCR-5 mutations do exhibit other clinical abnormalities, and so disorders, but importantly, this is
} not due to the HIV-1 virus. Rather, it is due to the genetic mutation. 
precisely because it is rooted in an infectious disorder composed of SARS-CoV-2 viruses disposed to be transmitted to other potential hosts.

We have throughout made reference to "hosts" of pathogens and infectious entities. In IDO Core instances of host bear a host role, which is a role borne by an acellular structure containing a distinct material entity, or organism whose extended organism contains a distinct material entity, realized in use of that structure or organism as a site of reproduction or replication. Reference to acellular structure accommodates the case of a virus that serves as host to an infecting virophage. Our definition also provides the resources needed to characterize the implicit temporal ordering in the definition of infectious disposition represented in Figure 2.

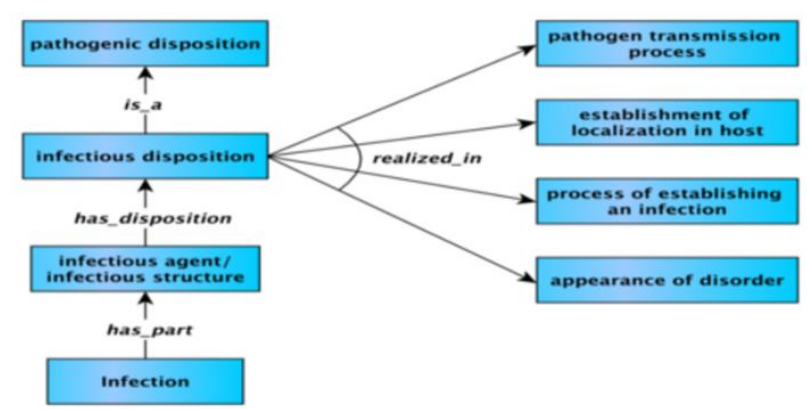

Figure 2 | Some aspects of IDO infectious disposition

When an infectious entity realizes its infectious disposition, it is first transmitted to the host before establishing localization in the host, after which it will become part of an infection prior to the appearance of disorder. If the virus establishes itself in the host, becomes part of an infectionand that infection is causally linked to an elevated risk of either pain or other feelings of illness, or of death or dysfunction in the host — then the infection is also an infectious disorder, and so the host is disposed to undergo various pathological processes. But infection may also occur without such clinical abnormality, in which case the virus has failed to fully realize its infectious disposition, and so has failed to establish an infectious disorder.

Before turning to pathogen transmission in more detail, two points are worth making here. First, our definitions do not count the presence of commensal microflora in our microbiome, many of which bear an infectious disposition, as constituting either an infection or an infectious disorder. This is because the microbiome part of our extended organism is not formed by an invasive process of establishing an infection. Here we have only colonization of the host. Under normal circumstances the relevant pathogens are unable to realize their infectious disposition. Yet they can still form disorders in their hosts, if they end up in the wrong anatomical site, as in the case of bacteremia, or if a colony grows out of control, as in the case of yeast infections. Each of these cases involves an opportunistic pathogen, defined in IDO Core as a pathogen with an opportunistic infectious disposition, in turn defined as an infectious disposition to become part of a disorder only in organisms whose defenses are compromised. ${ }^{2}$ Thus our definitions allow for the representation of infectious disorders caused by organisms that are typically commensal.

Second, the preceding definitions are accompanied by logical axioms used in querying and automated reasoning over the ontology. Figure 3, for example, illustrates how the axioms relating to infectious disorder entail, rightly, that it is an inferred subclass of infection.

\footnotetext{
${ }^{2}$ For example, commensal oral bacteria may infect the bloodstream following damage caused by vigorous brushing. Yeast overgrowth may occur when a healthy balance of defensive bacteria is reduced in the host.
} 


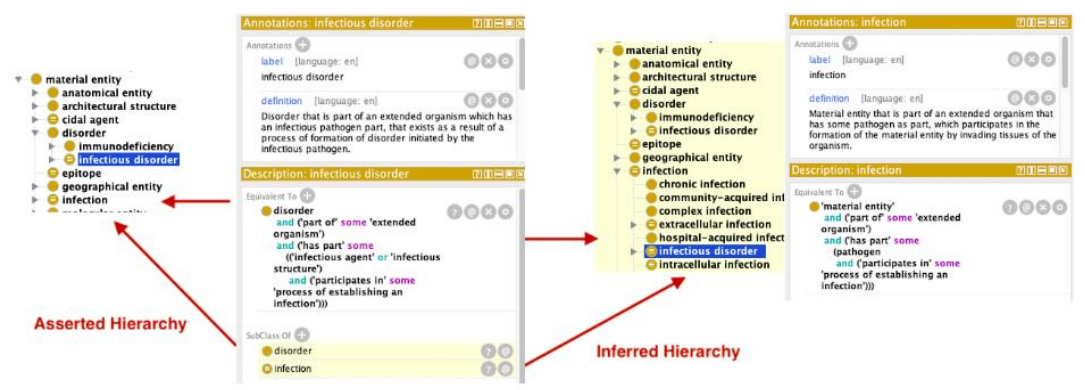

Figure 3 | Infectious Disorder inferred subclass of infection

\subsection{Transmission of Pathogens and Infectious Entities}

IDO Core characterizes infectious disease transmission in its various forms. From the Pathogen Transmission Ontology [20] IDO Core imports terms such $\mathrm{as}^{3}$ pathogen transmission process, which is a process during which a pathogen is transmitted directly or indirectly to a new host, and indirect pathogen transmission process, which is a pathogen transmission process in which a pathogen is indirectly transferred to a host by intermediary vehicles or vectors. Infectious diseases vary widely in associated transmission processes. For example, malaria and dengue fever are vector borne, while Schistosoma helminth parasites spend part of their life cycle within intermediate hosts after which they are transmitted into another medium, such as water, which then directly transmits the pathogen to definitive hosts such as humans. Table 4 reports IDO Core definitions relevant to a wide range of transmission types.

Table 4 I IDO Core transmission definitions

\begin{tabular}{||l||l||}
\hline \hline \multicolumn{1}{|c||}{ IDO Core Term } & \multicolumn{1}{c|}{ Definition } \\
\hline \hline pathogen transporter role & $\begin{array}{l}\text { Role borne by a material entity in or on which a pathogen is } \\
\text { located, from which the pathogen may be transmitted to a new } \\
\text { host. }\end{array}$ \\
\hline \hline pathogen vector role & $\begin{array}{l}\text { Pathogen transporter role that is borne by an organism active } \\
\text { in the transfer of an infectious agent or infectious structure to } \\
\text { an organism of another species in which it can realize its } \\
\text { infectious disposition. }\end{array}$ \\
\hline \hline host role & $\begin{array}{l}\text { Role borne by an acellular structure containing a distinct } \\
\text { material entity, or organism whose extended organism contains } \\
\text { a distinct material entity, realized in use of that structure or } \\
\text { organism as a site of reproduction or replication. }\end{array}$ \\
\hline \hline pathogen host role & $\begin{array}{l}\text { Host role borne by an organism having a pathogen as part of } \\
\text { its extended organism. }\end{array}$ \\
\hline \hline symbiont host role & $\begin{array}{l}\text { Host role borne by an organism whose extended organism } \\
\text { provides an environment supportive for the survival, growth, } \\
\text { maturation, or reproduction of an object contained as a proper } \\
\text { part. }\end{array}$ \\
\hline
\end{tabular}

While the definition of pathogen transporter role requires the bearer to actually have a pathogen located in or on it, bearers also have certain dispositions that enable them to play this role. While a mosquito bears the pathogen vector role only when a malaria parasite is located in or on it, there also inheres in its

${ }^{3}$ For IDO Core we have modified the textual definitions from their originals to align with BFO principles [2]. 
physical structure a disposition to transfer the parasites, which it has whether or not it contains any parasites. Similarly, for respiratory droplets that serve as vehicles for viruses such as SARS CoV-2. Notice also that a mosquito plays the vector role even when it is actively transferring a malaria parasite to a non-infectable human being bearing the sickle-cell trait. What is important is that the parasite is being transferred to an organism of a species in which its infectious disposition can typically be realized. A mosquito is not playing the vector role when transferring the parasite to an organism of a non-susceptible species.

The preceding selection does not exhaust those host roles included in IDO Core but does reflect the wide range of ways in which to characterize host-symbiont relationships.

\subsection{Pathogen Inhibition and Control}

IDO Core provides terms relevant to treatment agents, such as cidal agent and static agent. The former is the bearer of a cidal agent disposition, a disposition realized in the killing of bacteria, fungi, parasites, or viruses. The latter is the bearer of a static agent disposition, a disposition realized in a process of inhibiting the reproduction of bacteria, fungi, or parasites, or a process of inhibiting the replication of viruses. Subclasses of cidal agent disposition, such as bactericidal disposition (disposition to kill bacteria) and viricidal disposition (disposition to kill viruses), as well as cidal agent subclasses_-such as bactericidal and viricide - are defined in the corresponding pathogen-type IDO reference ontologies (see 2.1). The same for pathogen-type subclasses of static agent disposition and static agent. Agents that target only specific bacterial, fungal, parasitic, or viral species are to be defined in IDO extensions for the specific pathogens.

By our definitions the immune system, and the cells and cellular entities that constitute it, bear both cidal and static agent dispositions (as do devices such as autoclaves and sterilizers). Many drugs work not by directly killing or inhibiting pathogens, but rather by ramping up the immune system. While many associate terms like bactericidal and viricide with drugs and other chemical substances, researchers also use such terms to describe proteins in the immune system, especially interferongamma which is secreted by $\mathrm{T}$ helper cells.

Related is another notable aspect of IDO, which is its treatment of the phenomenon of resistance [21]. Examples include a population's herd immunity to certain infectious agents and the resistance of certain pathogens to antimicrobial drugs. IDO Core characterizes this phenomenon as involving an entity bearing protective resistance, a disposition that inheres in the entity by virtue of it having some part which is disposed to mitigate damage to the entity. For instance, a host's immunity to a given virus is a type of protective resistance. The host has certain parts, such as immune cells, that are disposed to secrete antibodies, neutralizing viral particles, and preventing the virus from infecting the host. Protective resistance is further characterized in terms of a "blocking disposition" [11, 21], a disposition the manifestation of which prevents, or mitigates, the realization of another disposition. Thus, the disposition of a host's immune cell acts as a blocking disposition since the process of antibody secretion prevents the virus from realizing its own disposition to infect and cause damage to the host.

We have refined the definition of protective resistance to narrow its scope, now defining it as a "Disposition inhering in an acellular structure or organism, with a part having a disposition to mitigate damage to the entity from internal and invasive threats, which is realized in one or more negative biological regulation processes." The last clause refers to the GO class negative regulation of biological process, a process that stops, prevents, or reduces the frequency, rate or extent of a biological process. Thus, my blocking of a knife thrust is not the realization of a protective resistance, as a knife thrust is not a biological process. When, in contrast, a virus evades a host immune response (a biological process) it is realizing a protective resistance. A related case study is provided in Additional File 3. 


\subsection{Epidemiology and Surveillance}

IDO Core includes terms for population-level processes, such as the epidemiological spread of disease as represented in Figure 4.

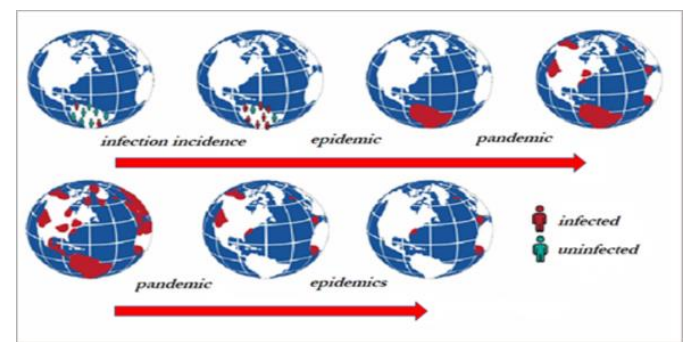

Figure 4 | Transitions through epidemic and pandemic

When an infection incidence in a population increases beyond a certain threshold in a geographic region, this may signal an epidemic in the region. When epidemics emerge in distinct geographic regions, this may signal the emergence of a pandemic. Over time, a pandemic may involve more or fewer geographic regions, and remain a pandemic. However, once the number of epidemics decreases below a certain threshold, there is no longer a pandemic. Similarly, the distribution of infections among members of a population may change while sustaining an epidemic, but once the infection incidence falls below a certain threshold, there is no longer an epidemic. IDO Core terms in Table 5 provide resources needed to represent these phenomena.

Table 5 | IDO Core epidemiological terms

\begin{tabular}{|c|c|}
\hline IDO Core Term & Definition \\
\hline infectious disease epidemic & $\begin{array}{l}\text { Process of infectious disease realizations for which } \\
\text { there is a statistically significant increase in the } \\
\text { infectious disease incidence of a population. }\end{array}$ \\
\hline infectious disease pandemic & $\begin{array}{l}\text { Process in which multiple infectious disease epidemics } \\
\text { of the same type of infectious disease unfold over } \\
\text { overlapping periods of time and affect organism } \\
\text { populations located in different geographic regions, } \\
\text { including different countries and continents. }\end{array}$ \\
\hline infectious disease incidence & $\begin{array}{l}\text { Quality that inheres in an organism population and is } \\
\text { the number of realizations of an infectious disease for } \\
\text { which the infectious disease course begins during a } \\
\text { specified period of time. }\end{array}$ \\
\hline directive information content entity (IAO) & $\begin{array}{l}\text { Information Content Entity that consists of a set of } \\
\text { propositions or images (as in the case of a blueprint) } \\
\text { that prescribes some Entity. }\end{array}$ \\
\hline $\begin{array}{l}\text { disease surveillance objective specification } \\
(A P O L L O-S V)\end{array}$ & $\begin{array}{l}\text { Objective specification whose endpoint is human } \\
\text { awareness of the level of a disease in a particular } \\
\text { population of a given biological taxon during some time } \\
\text { interval. }\end{array}$ \\
\hline $\begin{array}{l}\text { infectious disease control objective } \\
\text { specification (APOLLO-SV) }\end{array}$ & $\begin{array}{l}\text { Objective specification that is realized by processes that } \\
\text { are able or likely to stop the spread of a disease in a } \\
\text { population. }\end{array}$ \\
\hline infectious disease control strategy & Plan specification whose objective specification is an \\
\hline
\end{tabular}




\begin{tabular}{||l||l||}
\hline \hline APOLLO-SV) & infectious disease control objective specification. \\
\hline \hline contact tracing (APOLLO-SV) & $\begin{array}{l}\text { Infectious disease control strategy that identifies and } \\
\text { treats contacted organisms in a host population. }\end{array}$ \\
\hline \hline quarantine control strategy (APOLLO-SV) & $\begin{array}{l}\text { Infectious disease control strategy whereby } \\
\text { asymptomatic carriers who have had contact with } \\
\text { pathogens are prevented from having contact with } \\
\text { other susceptible organisms. }\end{array}$ \\
\hline
\end{tabular}

In addition to infectious disease incidence, IDO Core includes other qualities of infected populations, such as infectious disease mortality rate and infectious disease endemicity. IDO Core's coverage of epidemiology has been enhanced with a variety of term imports from the Apollo Structured Vocabulary (Apollo-SV) [22], which provides a standardized vocabulary for terms and relations required for the interoperation between epidemic simulator models and public health application software that interface with these models. Apollo-SV draws heavily on the Information Artifact Ontology (IAO) [23], and the terms in Table $\mathbf{5}$ which have been imported to IDO Core from Apollo-SV are subclasses of the IAO class directive information content entity. IDO Core has also been expanded with new classes, including pathogen surveillance and vector surveillance. The former are surveillance processes aiming to produce information about one or more pathogens with the purpose of managing those pathogens, while the latter are surveillance processes aiming to produce information about changes in the geographical distribution and density of one or several pathogen vectors with the purpose of facilitating appropriate and timely decisions regarding interventions.

\section{Extensions of IDO Core}

IDO Core is a hub from which a variety of spoke ontologies covering specific infectious diseases extend. Table 6 provides a list of the IDO Core extensions at their current state of development. Details of these ontologies can be found within Additional File 1 in Supplementary Table 2 and Supplementary Table 4. Other disease ontologies employing IDO terms are discussed in Supplementary Table 3. Supplementary Table 5 and Supplementary Table 6 detail databases and other applications to which IDO Core and its extensions have been applied.

Table 6 | IDO Extension Ontologies

\begin{tabular}{||l||l||}
\hline \hline $\begin{array}{l}\text { Coronavirus Infectious Disease Ontology (CIDO) } \\
{[24-26]}\end{array}$ & $\begin{array}{l}\text { Most recent version uploaded to Bioportal on } \\
\text { May 9, 2021 [27] }\end{array}$ \\
\hline \hline Influenza Ontology (IDOFLU) [28] & $\begin{array}{l}\text { Most recent version uploaded to BioPortal on } \\
\text { August 20, 2015 [29] }\end{array}$ \\
\hline \hline Brucellosis Ontology (IDOBRU) [30] & $\begin{array}{l}\text { Most recent version uploaded to BioPortal on } \\
\text { March 28, 2015 [3]] }\end{array}$ \\
\hline \hline IDO Virus (VIDO) [32] & $\begin{array}{l}\text { Most recent version uploaded to BioPortal on } \\
\text { August 3, 2020 [33] }\end{array}$ \\
\hline \hline $\begin{array}{l}\text { COVID-19 Infectious Disease Ontology (IDO- } \\
\text { COVID-19) [32] }\end{array}$ & $\begin{array}{l}\text { Most recent version uploaded to BioPortal on } \\
\text { August 3, 2020 [34] }\end{array}$ \\
\hline \hline Dengue Fever Ontology (IDODEN) [35] & $\begin{array}{l}\text { Most recent version uploaded to BioPortal on } \\
\text { February 17, 2014 [36] }\end{array}$ \\
\hline \hline Malaria Ontology (IDOMAL) [37] & $\begin{array}{l}\text { This ontology is obsoleted but is being hosted } \\
\text { for legacy purposes [38]. }\end{array}$ \\
\hline \hline Meningitis Ontology (IDOMEN) [39] & $\begin{array}{l}\text { Draft version uploaded on November 27, 2019 } \\
{[40]}\end{array}$ \\
\hline
\end{tabular}




\begin{tabular}{||l||l||}
\hline \hline Plant Disease Ontology (IDOPlant) [41] & Draft version released in 2012 [42] \\
\hline \hline $\begin{array}{l}\text { Staphylococcus aureus Infectious Disease } \\
\text { Ontology (IDOSA) [11, 20, 43] }\end{array}$ & Released on June 22, 2012 [44] \\
\hline \hline Schistosomiasis Ontology (IDOSCHISTO) [45] & Most recent version uploaded on October 23, \\
& $2013[46]$ \\
\hline \hline HIV Ontology (IDOHIV [47] & $\begin{array}{l}\text { Most recent version uploaded to Bio Portal on } \\
\text { April 4, 2017 [48] }\end{array}$ \\
\hline
\end{tabular}

Ideally, all IDO Core extension ontologies would be developed in the same way, and in conformance to all Foundry principles. Unfortunately, not all of the Foundry principles have been followed faithfully by the IDO Core extension ontologies represented in Table 6. Surveying extensions of IDO Core revealed a range of issues in these extensions, which are detailed at length, alongside recommendations for correction, in Additional File 2.

\subsection{Partitioning the IDO Suite and Creating a Lattice of Infectious Disease Ontologies} While currently existing IDO extensions were designed as direct extensions of IDO Core, several extension ontologies have defined terms which are not included in IDO Core, but which are useful where a group of extension ontologies cover the same pathogen type. For example, the term viriona single complete virus particle - is needed for each IDO Core extension covering viral infectious diseases, but is not needed in, say, representations of fungal infectious diseases. These observations suggest the need for pathogen-type specific reference ontology extensions of IDO Core. IDO Core extensions can easily be partitioned into subgroups based on pathogen type. For example, CIDO and IDOFLU both cover infectious viral diseases while IDOBRU and IDOSA both cover infectious bacterial diseases.

Additionally, the range of issues identified in Additional File 2 provides motivation for coordinated partitioning of the IDO suite of ontologies. IDO Core extensions were often developed without sustained coordination with nearby extensions. While we discuss how we intend to address such coordination issues below, for our purposes here, we note that creating pathogen-type specific reference ontology extensions of IDO Core creates fewer opportunities for misalignment among extensions. Much like a researcher who seeks to represent influenza can rely on IDO Core as a reliable starting point, and so not need to reflect on what exactly, say, an "infectious disease" is, similarly the same researcher importing a virus-specific extension of IDO Core would not need to reflect on what exactly, say, a "virus" is.

Grouping IDO Core extensions based on pathogen type is coordinated by the development of reference ontologies comprised of terms common to scientific investigations of the relevant pathogen. The resulting ontologies themselves extend directly from IDO Core and provide a hub from which pathogen-specific ontologies extend. Partitioning IDO Core extensions based on pathogen type results in bacteria, virus, fungi, and parasite specific reference ontologies, as illustrated in Figure 5. IDOSA, IDOMEN, IDOTB, IDOIE and IDOBRU extend from IDO Bacteria. IDOFLU, IDOHIV, IDODEN and CIDO extend from IDO Virus, while IDOSCHISTO and a new ontology for malaria (replacing IDOMAL) extend from IDO Parasite. 


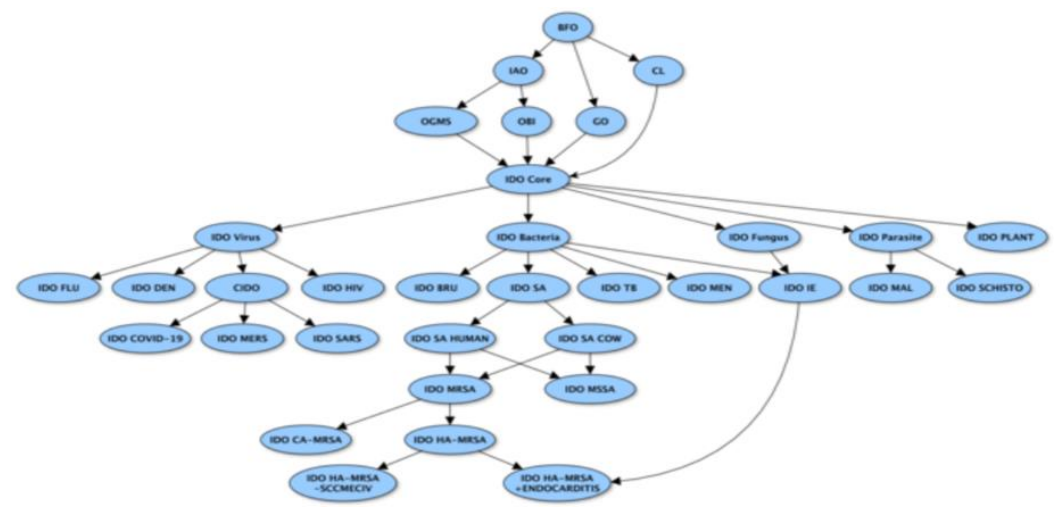

Figure 5 | A lattice of infectious disease ontologies

[43] shows how IDOSA annotations of genetic, phenotypic, and demographic data on S. aureus isolates maintained by the Network on Antimicrobial Resistance in Staphylococcus aureus [49] can be used to infer lattice application ontologies for specific subfamilies of $S$. aureus-related diseases, down to the level of specific strains. The method is generalizable to isolate repositories across the infectious disease domain. Leveraging the other extension ontologies within the IDO suite, the method allows us to generate similar lattices for specific subfamilies of coronavirus-related diseases, influenza virus-related diseases, and so on. Together these form a larger network of infectious disease ontologies under IDO Core as illustrated in Figure 5.

In this figure, where two ontologies are connected by an arrow, the one lower in the lattice extends, and imports needed terms from, the latter, as well from other ontologies higher up. To be clear, subontologies only import what is needed, not all of the terms and axioms from all the ontologies of which it draws. The ontologies at the very top are upper-level OBO ontologies from which IDO Core, and other ontologies further down in the lattice, extend. Note that the graph presents only a representative sample, rather than an exhaustive list, of upper-level ontologies upon which the lattice depends.

The remainder of our discussion focuses on a recent partitioning of the IDO suite of viral infectious disease ontologies under the Virus Infectious Disease Ontology (VIDO) and extensions covering coronavirus infectious diseases. We intend the work described below to serve as a model for the re-engineering of existing IDO Core extensions in such a way as to yield greater conformance with the ontology building principles discussed in the foregoing.

\subsection{Virus Infectious Disease Ontology}

VIDO [32] is a virus-neutral extension of IDO Core including terminological content used by researchers across various domains interested in the study of viral infectious diseases. VIDO thus provides a common language for IDO Core extensions covering viral infectious diseases such as IDOFLU and CIDO. Our example extensions from VIDO will focus on coronaviruses.

Like other IDO Core extensions, VIDO introduces terms from existing OBO Foundry ontologies where needed, such as OBI, NCBITaxon, and many others. From the NCBITaxon VIDO imports the term virus and asserts it to be a subclass of acellular structure. VIDO also imports lower-level subclasses of virus from the NCBITaxon representing entities investigated by virologists such as prion, viroid, and satellite.

The NCBITaxon provides an exhaustive list of life science terms. However, three issues are worth noting when reusing NCBITaxon terms: First, with respect to virus terms NCBITaxon appears to align with the widely used International Committee on Taxonomy of Viruses (ICTV). However, ICTV guidance lacks systematic classification criteria and consequently leaves several 
viruses unclassified [50]. Second, when NCBITaxon is combined with automated importing tools such as the widely used Ontofox [51], this may result in the importing of an entire ICTV structured hierarchy - stretching from kingdom to species - resulting in large, unwieldy, taxonomies obscuring classes of interest. Third, NCBITaxon itself provides few textual definitions for terms. To align with OBO Foundry metadata conventions [52] and best practices [2], textual definitions and logical axioms are needed for virus and subclasses.

These issues suggest that imported NCBITaxon terms should be supplemented with a more robust, simpler, ontological structure with accompanying textual definitions. The Baltimore Classification of viruses [53] - which groups viruses based on features of genetic structure addresses both concerns, yielding seven, exhaustive, classes we import from the NCBITaxon as subclasses of virus corresponding to the Baltimore Classification.

Figure 6 illustrates the Baltimore Classification in Protégé, supplemented by a standard visual summary of the seven viral replication pathways underwritten by virus genetic differences.

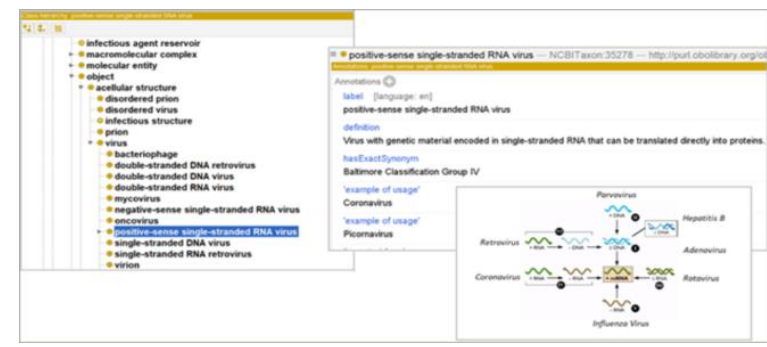

Figure 6 | Protégé representation of Baltimore Classification

More generally, VIDO using the Baltimore Classification provides developers of more specific virus ontologies needed textual definitions, and a succinct, navigable, ontological structure which refers to viral replication pathways, and so the obligate pathogenicity of viruses.

The IDO Core classes infectious disorder, disease, and disease course provide parent classes from which virus-specific children can be defined, as represented in Table 7 illustrating a simple recipe for extending IDO Core to a more specific domain.

Table 7 Virus and subclass definitions from VIDO

\begin{tabular}{||l||l||}
\hline \multicolumn{1}{|c|}{ Term } & \multicolumn{1}{c||}{ Definition } \\
\hline \hline virus & $\begin{array}{l}\text { Acellular structure with RNA or DNA genetic material which } \\
\text { uses host metabolic resources for RNA or DNA replication. }\end{array}$ \\
\hline \hline $\begin{array}{l}\text { positive-sense single-stranded } \mathbf{R N A} \\
\text { virus }\end{array}$ & $\begin{array}{l}\text { Virus with genetic material encoded in single-stranded RNA } \\
\text { that can be translated directly into proteins. }\end{array}$ \\
\hline \hline virus disorder & $\begin{array}{l}\text { Infectious disorder that exists as a result of a process of } \\
\text { formation of disorder initiated by a virus. }\end{array}$ \\
\hline \hline viral disease & $\begin{array}{l}\text { Infectious disease inhering in a virus disorder that is a } \\
\text { disorder due to the presence of the virus. }\end{array}$ \\
\hline \hline \multirow{2}{*}{ viral disease course } & $\begin{array}{l}\text { Infectious disease course whose physical basis is a virus } \\
\text { disorder that is clinically abnormal in virtue of the presence } \\
\text { of the relevant virus. }\end{array}$ \\
\hline \hline \multirow{2}{*}{ symptomatic carrier role (IDO Core) } & $\begin{array}{l}\text { Pathogen host role borne by an organism whose extended } \\
\text { organism contains a pathogen bearing an infectious } \\
\text { disposition towards the host, and the host has manifested } \\
\text { symptoms of the infectious disease caused by the pathogen. }\end{array}$ \\
\hline
\end{tabular}




\begin{tabular}{||l||l||}
\hline \hline asymptomatic carrier role (IDO Core) & $\begin{array}{l}\text { Pathogen host role borne by an organism whose extended } \\
\text { organism contains a pathogen bearing an infectious } \\
\text { disposition towards the host, and the host has no symptoms } \\
\text { of the infectious disease caused by the pathogen. }\end{array}$ \\
\hline \hline subclinical infection (IDO Core) & Infection that is part of an asymptomatic carrier. \\
\hline \hline subclinical virus infection & Subclinical infection that is part of a virus host. \\
\hline \hline
\end{tabular}

A given virus disorder is a material basis of some associated viral disease which may be realized in some associated viral disease course. Symptomatic cases of virus infection can be represented by importing terms from the Symptom Ontology, such as dry cough, fever, taste alteration, smell alteration, among others [54]. Worth noting is that these definitions are compatible with, for example, counting an asymptomatic carrier of SARS-CoV-2 as having the associated disease. This result aligns, moreover, with the CDC's case criteria adopted on April $5^{\text {th }}, 2020$ which indicates that the presence of the SARS-CoV-2 genome or relevant antigens in an individual is sufficient to count as a case of COVID-19, and that asymptomatic cases should be counted as instances of the disease $[55,56]$.

Indeed, IDO Core already provides terms useful for distinguishing symptomatic and asymptomatic virus carriers, as well as subclinical infections from clinical infections, with relevant terms found in Table 7. The term subclinical infection reflects standard - if somewhat obscure - use of the terms "subclinical" and "asymptomatic" while nevertheless allowing for cases in which hosts with clinically abnormal infections exhibit no symptoms. For VIDO, this term is straightforwardly extended to subclinical virus infection, which is an infection caused by a virus that is part of an asymptomatic carrier.

\subsection{The Coronavirus Infectious Disease Ontology}

VIDO was developed as a bridge between IDO Core and extension ontologies representing specific diseases and specific causative pathogens. An extension of importance during the pandemic is the recently developed CIDO. Developed by Oliver He and his team, CIDO provides semantic resources needed for representing coronavirus genome, surveillance, vaccine, and host data. CIDO has been used to annotate 136 known anti-coronavirus drugs [57], identify 110 candidate drugs [23] for COVID-19 drug repurposing [58], and provides input to machine learning efforts [24] in identifying potential COVID-19 vaccines. Several members of both the IDO and VIDO development teams are also members of the CIDO development team working to ensure alignment among these ontologies, and adherence to OBO Foundry principles. Like VIDO, CIDO imports terms from a wide range of ontologies, including IDO Core, ChEBI [59], UBERON [60], GO, the Vaccine Ontology [61], and the NCBITaxon.

CIDO can straightforwardly extend from VIDO by adopting terms such as those in Table 8. More generally, CIDO can be populated by starting with a given virus term from VIDO, and then creating a subclass of that term restricted to members of the species coronavirus and associated diseases. Following representation of the Baltimore Classification in VIDO, for example, a subclass for positive-sense single-stranded RNA virus reflecting instances of coronavirus which can be imported from the NCBITaxon, and for which a definition was generated above. Moreover, terms reflecting common features of coronaviruses can be imported from other OBO ontologies to characterize the virus species, such as that the viral genome including a five-prime nucleotide cap, or the common glycoprotein spikes found in the viral envelope [62, 63], many of which are represented in the Protein Ontology with terms such as SARS-CoV-2 membrane protein and SARS-CoV-2 spike glycoprotein. 
Table 8 Extension of CIDO from VIDO

\begin{tabular}{||l||l||}
\hline \multicolumn{1}{|c||}{ Term } & \multicolumn{1}{c||}{ Definition } \\
\hline \hline coronavirus & $\begin{array}{l}\text { Positive-sense single-stranded RNA virus with a } \\
\text { helically symmetrical nucleocapsid, lipid bilayer viral } \\
\text { envelope, and surface spike peplomers. }\end{array}$ \\
\hline \hline coronavirus disorder & $\begin{array}{l}\text { Virus disorder that exists as a result of a process of } \\
\text { formation of disorder initiated by a coronavirus. }\end{array}$ \\
\hline \hline coronavirus disease & Viral disease inhering in a coronavirus disorder. \\
\hline \hline coronavirus disease course & $\begin{array}{l}\text { Viral disease course that is the realization of some } \\
\text { coronavirus disease and has as a participant a } \\
\text { coronavirus. }\end{array}$ \\
\hline \hline
\end{tabular}

CIDO deals with coronavirus infectious diseases in general, and in that respect is more specific than VIDO. There are, however, several species of coronavirus which cause distinct infectious diseases, such as SARS-CoV-2 as the causative virus of COVID-19 and MERS-CoV as the causative virus of Middle-Eastern Respiratory Syndrome. Conformance with OBO guidelines requires ontologies be comprised of a small set of self-contained, reusable, terms and not unnecessarily duplicate terms found in other ontologies. There is a need in the present COVID-19 pandemic for terms specific to SARS-CoV-2 and COVID-19.

\subsection{The COVID-19 Infectious Disease Ontology}

IDO-COVID-19 extends from CIDO and covers COVID-19 and its cause SARS-CoV-2. IDOCOVID-19 thus brings together IDO Core, VIDO, and CIDO in the interest of fine-grained representation of this virus strain and associated diseases. Figure 7 summarizes links among these ontologies.

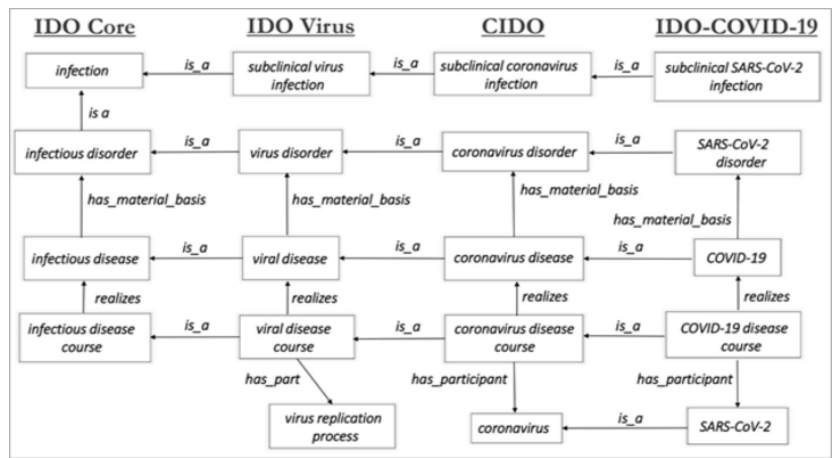

Figure 7 | Links between VIDO, CIDO and IDO-COVID-19

The starting point for IDO-COVID-19 is pathogenesis to COVID-19 caused by SARS-CoV-2. Flexibly representing COVID-19 pathogenesis is of importance during the current pandemic, as researchers are still working to understand how SARS-CoV-2 infections cause such a wide range of signs and symptoms across demographics. Representing COVID-19 pathogenesis in IDO-COVID19 requires importing relevant terms from VIDO, CIDO, and relevant OBO Foundry ontologies, to define terms such as those found in Table 9. Instances of $S A R S-C o V-2$ pathogenesis are in turn asserted as part of some COVID-19 disease course.

Table 9 | Extension of IDO-COVID-19 from CIDO 


\begin{tabular}{|c|c|}
\hline Term & Definition \\
\hline COVID-19 & "Coronavirus disease inhering in a SARS-CoV-2 disorder. \\
\hline COVID-19 disease course & $\begin{array}{l}\text { Coronavirus disease course that is the realization of some } \\
\text { COVID-19 disease and has participant SARS-CoV- } 2 \text {. }\end{array}$ \\
\hline pathogenesis (GO) & $\begin{array}{l}\text { Process that generates the ability of a pathogen to induce } \\
\text { disorder in an organism. }\end{array}$ \\
\hline coronavirus pathogenesis (CIDO) & $\begin{array}{l}\text { Coronavirus process realization of an infectious disposition } \\
\text { inhering in a coronavirus or coronavirus population, have at } \\
\text { least the process parts: (1) pathogen transmission, (2) } \\
\text { establishment of localization in host, (3) process of } \\
\text { establishing an infection, and (4) appearance of a virus } \\
\text { disorder. }\end{array}$ \\
\hline SARS-CoV-2 pathogenesis & $\begin{array}{l}\text { Coronavirus pathogenesis process realization of an } \\
\text { infectious disposition inhering in SARS-CoV-2 or a SARS- } \\
\text { CoV-2 population, having at least the proper process parts: } \\
\text { (1) pathogen transmission, (2) establishment of localization } \\
\text { in host, (3) process of establishing an infection, and (4) } \\
\text { appearance of a virus disorder. }\end{array}$ \\
\hline replication (IDO Core) & $\begin{array}{l}\text { Production process in which a participant creates a copy of } \\
\text { itself. }\end{array}$ \\
\hline virus replication (VIDO) & $\begin{array}{l}\text { Replication process in which a virus containing some } \\
\text { portion of genetic material inherited from a parent virus is } \\
\text { replicated. }\end{array}$ \\
\hline generative stage (IDO Core) & Temporal subdivision of a developmental process. \\
\hline virus generative stage (VIDO) & $\begin{array}{l}\text { Infectious structure generative stage that is a temporal } \\
\text { subdivision of a virus developmental process. }\end{array}$ \\
\hline virus attachment stage (VIDO) & $\begin{array}{l}\text { Virus generative stage during which a virion protein binds to } \\
\text { molecules on the host surface or host cell surface } \\
\text { projection. }\end{array}$ \\
\hline "virus penetration stage (VIDO) & $\begin{array}{l}\text { Virus generative stage during which a virion or viral nucleic } \\
\text { acid breaches the barriers of a host. }\end{array}$ \\
\hline SARS-CoV-2 attachment stage & $\begin{array}{l}\text { Virus attachment stage during which SARS-CoV-2 bonds } \\
\text { with a host cell. }\end{array}$ \\
\hline SARS-CoV-2 penetration stage & $\begin{array}{l}\text { Virus penetration stage during which SARS-CoV-2 } \\
\text { penetrates a host cell. }\end{array}$ \\
\hline SARS-CoV-2 adhesion susceptible cell & $\begin{array}{l}\text { Virus adhesion susceptible cell with a functional receptor } \\
\text { part bearing an adhesion disposition realized in a SARS- } \\
\text { CoV-2 attachment stage. }\end{array}$ \\
\hline SARS-CoV-2 penetration disposition & $\begin{array}{l}\text { Virus penetration disposition borne by a functional receptor } \\
\text { complex that is the disposition to participate in a SARS- } \\
\text { CoV-2 penetration process. }\end{array}$ \\
\hline $\begin{array}{l}\text { negative regulation of SARS-CoV-2 } \\
\text { attachment }\end{array}$ & $\begin{array}{l}\text { Negative regulation of coronavirus replication process that } \\
\text { stops, prevents, or reduces the frequency of some SARS- } \\
\text { CoV-2 attachment stage. }\end{array}$ \\
\hline $\begin{array}{l}\text { negative regulation of } S A R S-C o V-2 \\
\text { penetration }\end{array}$ & $\begin{array}{l}\text { Negative regulation of coronavirus replication that stops, } \\
\text { prevents, or reduces the frequency of some SARS-CoV- } 2 \\
\text { penetration stage. }\end{array}$ \\
\hline
\end{tabular}


The term coronavirus pathogenesis will ultimately be imported from CIDO, and is itself a subclass of the VIDO term viral pathogenesis, a subclass of pathogenesis imported from the Gene Ontology. As defined, pathogenesis is a success term, in that it encompasses formation of disorder in an entity. This is reflected in (1)-(4) of the SARS-CoV-2 pathogenesis definition and motivated by the GO Consortium focus on canonical biological processes [4]. This is not to say all SARS-CoV-2 infections result in successful pathogenesis. An individual may be infected by SARS-CoV-2, but this need not result in a relevant disorder. Absent the relevant disorder, there is no appropriate material basis for COVID-19. Consequently, this would not count as an instance of SARS-CoV-2 pathogenesis, as the process part (4) would be missing.

Instances of viral disease course and virus pathogenesis have as respective parts virus replication. SARS CoV-2 pathogenesis clearly involves replication in a host. The term virus replication is defined in VIDO as a subclass of the IDO Core term replication. IDO-COVID-19 imports the newly minted generative stage from IDO Core, defined as a temporal subdivision of a developmental process. Subclasses of which include the various stages through which viruses may proceed during a given replication.

Not all cells are susceptible to SARS-CoV-2 infection. In those cases of successful infection, the virus attaches to the alveolar epithelial cell with a spike surface glycoprotein, by way of these host cell's angiotensin-converting enzyme 2 (ACE2) receptors [64, 65]. ACE2 receptors appear crucial for SARS-CoV-2 attachment, suggesting the need to define SARS-CoV-2 adbesion susceptible cell, which is a cell bearing an adhesion disposition realized in a $S A R S-C o V-2$ attachment stage, where the functional receptor material base ACE2 is imported to IDO-COVID-19 from the Protein Ontology [66] (from which it also imports recently created terms for SARS-CoV-2 proteins). A SARS-CoV-2 attachment stage is frequently followed by a penetration stage, involving penetration susceptible cells. More specifically, transmembrane protease serine 2 (TMPRSS2) aids in cleaving host cells in anticipation of SARS-CoV-2 fusing with the cell membrane [67], then introducing viral genomic RNA into the cytoplasm.

This similarly suggests a need to define $S A R S-C o V-2$ penetration susceptible cells as cells bearing a $S A R S-C o V-2$ penetration disposition where in this case the functional receptor material base is TMPRSS2, also imported to IDO-COVID-19 from the Protein Ontology. Reflection on other stages suggest corresponding terms, since following penetration, SARS-CoV-2 genome translation and virion assembly begins in the endoplasmic reticulum, forming virions then packaged into vesicles, sent to the host Golgi apparatus, and fused with the host cell membrane to exit the host. IDO-COVID-19 terms reflecting stages of the replication cycle for SARS-CoV-2 also provide targets for regulation of that cycle, important to vaccine, drug, and treatment options. Examples of negative regulation relevant here are negative regulation of $S A R S-C o V-2$ attachment and negative regulation of $S A R S-C o V-2$ penetration.

We should acknowledge that there are other ontology initiatives developed to support curation of COVID-19 data, such as the WHO COVID-19 Rapid Version CRF [68], the COVID-19 Surveillance Ontology [69], the Linked COVID-19 Data Ontology [70], and the NASA Jet Propulsion Laboratory's COVID-19 Research Knowledge Graph [71]. However, since each is a stand-alone initiative developed outside the scope of OBO Foundry principles, each is subject to the silo problems documented in the introduction.

\section{DISCUSSION}

Since IDO Core is built in accordance with the OBO Foundry principles, this means that the IDO ontologies are interoperable with other OBO Foundry ontologies. IDO Core, VIDO, CIDO, and IDO-COVID-19, for example employ a well-specified syntax, common identifiers, and a common top-level ontology, as required by the Foundry. Each is openly available in the public 
domain under creative commons licenses as well. Recognizing these ontologies are in neighboring domains, developers have worked closely to ensure each ontology is modular and remains within its clearly specified scope using unambiguous terms. Collaboration has taken the form of publications [32], conference presentations, and weekly harmonization meetings.

Ontology metadata can be used to combine heterogeneous bodies of research data to enable structured querying and analysis [72]. Figure 8 and Figure 9, illustrate, for example, simple Description Logic queries of IDO-COVID-19. The former returning any classes instances of which are mereological parts of virus replication processes, while the latter returns any class instances of which are preceded by some SARS-CoV-2 attachment stage. As has been revealed by the COVID19 pandemic, failure to pay heed to metadata standards limits the reusability of available primary genomic data, significantly impeding efficient response measures [73].

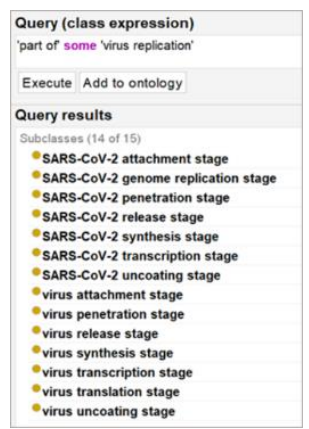

Figure 8 | DL Query for part_of some virus replication

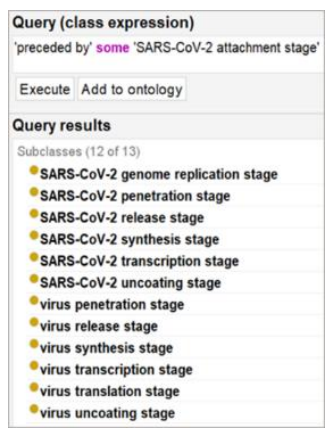

Figure 9|DL Query for preceded_by some $S A R S$ CoV-2 attachment stage

Adherence to Foundry principles makes the IDO ontologies applicable to the annotation of a variety of databases relevant to infectious disease that already make use of Foundry ontologies in their annotations [10]. For examples of databases to which IDO ontology annotations have been previously applied, see Supplementary Table 5.

VIDO, CIDO and IDO-COVID-19 are currently being used to annotate approximately 400 articles in the National Library of Medicine [74] COVID corpus, which report COVID-19 clinical trial, epidemiological, and pathogenesis data. The resulting 'gold standard' corpus will be used to train algorithms for automated annotating tasks. These algorithms will in turn be used to identify useful patterns in COVID-19 datasets on the model illustrated in [75], which describes a novel method for learning features of entities such as proteins and viruses from their associations to ontology classes, and describes how this method can be employed for fast identification of virushost interactions that can shed light on potential treatments and drug discoveries. That said, this work is in its infancy and we hope to report our results in future work.

In the ideal case data and information relevant to infectious disease research, independently of where they are stored, should be annotated using IDO terms. The resultant annotated data would thereby become available to computer processing as if they formed a single body of linked data in virtue of the semantically controlled properties of the IDO terms and of the logical structure of their definitions. Experience shows, however, that these benefits are difficult to achieve except in those cases where databases have been created using the ontology structure from the very beginning, an approach pursued most successfully in the case of the incorporation of Gene Ontology annotations into the UniProt database [76]. [77] provides an illustration of this approach in the field of influenza research. Matters are improving in this respect with the development of approaches to data 
annotation using machine learning. The results are still in many cases disappointing, but they are at least improving over time [78].

To accelerate these improvements, it will be necessary to associate with each OBO Foundry ontology a terminology comprising, for example, (1) those terms in common usage in the relevant literature that denote entities which are denoted by different terms in the ontology, (2) terms denoting entities that are more specific than are covered in the salient ontology. This will then require a special set of relationships to indicate, for any given term in the terminology, the nature of the annotation with an ontology term. Where ontology precedes data, annotation then becomes automatic.

All too often, however, problems arise, for example, because it is too difficult to associate terms from the controlled vocabulary with the terms used by those responsible for data collection. Terms in databases and literature may denote instances or types by using the exact same term that is used in an ontology to denote a perhaps related, but still different type. Furthermore, databases and literature may use terms that denote entities which in the ontology are denoted by different terms. Even more prevalent are terms in databases that denote entities more specific than those covered in an ontology. This requires a special set of relationships to indicate the nature of the annotation with the ontology term. In future work we hope to explore the extent to which the ontology structure of the IDO suite can enhance the construction of infectious disease databases by using the "ontology precedes data" approach employed with success by the Gene Ontology.

Admittedly, not all IDO extension ontologies have adequately adhered to the IDO strategy as presented in the foregoing. Part of the goal of our current work on CIDO, VIDO, and IDOCOVID-19 is to provide a model according to which other IDO extension ontologies can be brought into tighter coordination with the Core, as well as an easy-to-follow recipe for building new pathogen- specific ontologies so that infectious disease researchers are given fewer opportunities to generate inoperable ontologies. As we continue to face the threat of novel viruses (as well as bacteria and parasites) in the future, having such a blueprint in hand should facilitate more rapid extension of the IDO suite.

Relatedly, ontology annotations are all too often applied incorrectly. Many users of OBO Foundry ontologies do not seem to understand BFO, OGMS, or the principles upon which they are based. This suggests the OBO community needs to work harder to make sure these principles are well understood. And even where the principles are more or less understood, it is likely we need to be more vigilant in ensuring OBO Foundry users are actually complying with them. A complete solution to these issues is beyond the scope of this paper, though we intend our work here to illustrate some guidance to users working with OBO ontologies. Users should develop a firm understanding of the classes, relations, and principles of BFO, which can be fostered both by studying existing user guides [2,79] and online tutorials [80], and by signing up for and participating in the BFO user group [81]. Users should, moreover, develop competence in OBO methodological principles by reviewing the extensive guidance from the OBO Foundry website [6], Github issue tracker [82], and perhaps signing up for the OBO Slack channel [83] for discussion. Users should, additionally, understand the goals of ontology use, and how proper integration of a small, narrowly focused ontology, with other small ontologies, can result in significant semantic resources for existing domains and those yet to be represented. Internalizing the preceding information will provide a crucial foundation for the proper use of extensions of BFO in the OBO Foundry. Of course, users competent with $\mathrm{BFO}$ and $\mathrm{OBO}$ principles must be able to rely on other ontologists working with and building OBO ontologies. Ontologies that are counted in the OBO library which are not designed in conformance with $\mathrm{OBO}$ principles undermine OBO standards, and may lead to confusion among users. With that in mind, the OBO operations committee members and working group should play a more active role in ensuring OBO ontologies align with OBO principles. There 
should, perhaps, be stakes for allowing one's ontology to fall out of conformance with these standards, e.g. loss of membership in the library. Members of the OBO committees might schedule, for instance, routine inspections of ontologies to determine whether they align with OBO standards. From another direction, the OBO library might be given conformance or reliability ratings by committees, much like restaurants are given scores and organizations are given credit ratings. OBO already institutes something like this practice. There is presently the OBO Foundry - a small group of ontologies vetted by the more active members in the OBO community - and the broader OBO library - ontologies that met initial OBO standards for inclusion. Further tiers might be constructed so that users are more easily able to identify the paradigms of good ontology development, and consequently, use those ontologies to guide their own ontology development.

The lattice network, illustrated in section 2.1, can be used to define a strategy for constructing a taxonomy of infectious diseases incorporating both high- throughput genetic and molecular data as well as clinical data. The network can also be used for rapidly creating new ontologies for novel pathogens or novel strains in a way that provides a pathway for automatic linking of emerging data to legacy data relating to existing pathogens and diseases. The IDO suite of ontologies can thereby contribute to the advance of what is called 'personalized' or 'precision' medicine, which depends upon effective classification and association of biological disease data with known clinical phenotypes and disease types at ever finer levels of detail.

One might worry our lattice methodology may lead to a combinatorial explosion of ontologies. For example, the lattice of $S$. aureus infectious disease ontologies [43] suggests distinct ontologies will be needed for each strain, host, and so forth. In response, note application ontologies are added to the lattice if there is a need from researchers describing genuine biological phenomena, not simply because of combinatorial possibilities. Even so, one may worry that our lattice methodology coupled with advances in personalized medicine may lead to an explosion of personalized ontologies.

More specifically, representing individual patients may require fine-grained ontologies, with substantial overlap, and minor differences. In response, we find this a feature rather than a bug of our methodology. First, and again, if there is a need for personalized ontologies then we intend to be compatible with that need. Second, though personalized ontologies would perhaps overlap substantially, they will also be substantially distinct. For example, Sally and John both bear temperature as a determinable, but each bears a distinct temperature as a determinate quality. Similarly for mass, pathogen immunity, respiratory capacity, and so forth. Moreover, the token individual Sally will be distinct from the token individual John.

These remarks apply equally to newly emerging pathogens. For example, suppose we need a SARS-CoV-3-focused ontology. We then import from IDO Core, OBI, VIDO, CIDO and other ontologies, define what terminological content we can from imported terms. We introduce the virus SARS-CoV-3 as a primitive subclass of coronavirus. The result is an ontology largely composed of existing ontologies, with a proper part composed of combinations of that new primitive with existing terms - for example, SARS-CoV-3 infection, SARS-CoV-3 disorder, and so on. And, since by assumption, we need to represent SARS-CoV-3 data, we are justified in adding them. We should be as specific as researchers need.

Implementation of the lattice methodology requires significant maintenance and overhead to keep IDO Core, the mid-level ontologies, and the various sub-ontologies all in sync. If a definition or relationship changes in IDO Core, developers of extending ontologies will need to be notified. And if IDO Core changes in such a way that some of the axioms in downstream ontologies might become inconsistent, there should be some process by which this can be detected and resolved. Aware of such issues, we have created a Github Organization, where developers of IDO extension ontologies can discuss needs in the IDO ecosystem, coordinate together on updates, and are alerted to changes in ontologies populating the IDO organization [84]. The organization follows 
maintenance protocols modeled on OBO Foundry principles. While we do not at present implement any software tools to support automatic updates, we hope to explore the development of such tools in future work.

\section{Conclusions}

As we face the continued threat of novel pathogens in the future, IDO Core provides a simple recipe for building new pathogen-specific ontologies in a way that allows data about novel diseases to be easily compared, along multiple dimensions, with data represented by existing disease ontologies. The IDO strategy, moreover, supports ontology coordination, providing a powerful method of data integration and sharing that will allow physicians, researchers, and public health organizations to respond rapidly and efficiently both to the current and future public health crises.

\section{Methods}

With respect to editing tool, IDO Core was updated using the Protégé ontology development tool [85], leveraging the enhanced expressivity of the Web Ontology Language (OWL). Ontologies were tested against automated reasoners such as HermiT and Pellet. Additionally, logical axioms underwriting these ontologies were translated into a syntax readable by the Mace4 model checker, which allowed for manual graphical inspection of classes of models constrained by the asserted axioms. An automated proof-checker Prover9 bundled with Mace4 was used to validate expected theorems while refining axiom models.

IDO Core, like other OBO Foundry ontologies, is not exhaustive, as development of the ontology is intended to maintain pace with growing research on infectious diseases. With respect to updating IDO Core based on the existing OBO library, a study of extension ontologies was conducted in the interest of identifying terms in extensions that would be better placed in IDO Core. From another direction, a study of developments in OBO Foundry ontologies was conducted in the interest of identifying terms better suited to more general ontologies. In the event terms were needed for IDO Core which were not suitable for introduction, because too general for the domain of infectious diseases, term requests were made to developers of relevant OBO Foundry ontologies. For example, transmission classes were requested for and subsequently added to the Pathogen Transmission Ontology. Lastly, with respect to updating IDO Core based on the construction of novel reference ontologies, such as VIDO, collaborative study between IDO Core, VIDO, CIDO, and IDO-COVID-19 developers resulted in careful construction of relevant terms based on up-todate empirical literature, researcher term use, and logical coherence. For example, adjustments were needed to IDO Core's definition of infectious agent due to reflection on viruses, resulting in the introduction of the class acellular structure as parent class to virus.

In every case, terminological content for IDO Core was either imported from an existing OBO ontology, defined based on imported terms, introduced as a primitive to IDO Core, or defined based on IDO Core primitive and/or imported terms. In accordance with OBO Foundry principles, priority was given to importing and defining terms, over introducing primitive terms to IDO Core. Before new primitives were deemed necessary, IDO Core developers canvased researchers developing nearby ontologies for insights, posed queries on issue trackers on relevant GitHub pages, and studied relevant infectious disease literature. Terms were then introduced, vetted by specialists where possible, then introduced to IDO Core after scrutiny.

As with most $\mathrm{OBO}$ ontologies, IDO Core is an open project with its own GitHub repository [86], where the most recent published and developmental versions of the ontology are available for download. We encourage members of the ontology community, as well as infectious disease researchers, to submit term requests to our GitHub Issues tracker. The Issues tracker can also be 
used to report any errors or concerns related to the ontology. Before requesting a new term, please search online ontology repositories such as Ontobee and BioPortal to see if the needed term already exists. Once a term request is received, it will be reviewed by the main IDO Core team to determine whether the term is most appropriate for IDO Core, one of its extensions, or another OBO ontology. If the term is within IDO Core's scope, then it will be added with a formal definition, written in conjunction with the term requestor to ensure biological accuracy as well as adherence to OBO Foundry best practices and consistency with IDO logical structure. We can assign a unique ID for the term so that it can be used for immediate annotation prior to the definition being finalized.

\begin{abstract}
Abbreviations
Apollo-SV: Apollo Structured Vocabulary; BFO: Basic Formal Ontology; CIDO: Coronavirus Infectious Disease Ontology; ChEBI: Chemical Entities of Biological Interest; CL: Cell Ontology; GO: Gene Ontology; IDOBRU: Brucellosis Ontology; IDO Core; Infectious Disease Ontology Core; IDODEN: Dengue Ontology; IDOFLU: Influenza Ontology; IDOHIV: HIV Ontology; IDOMAL: Malaria Ontology; IDOMEN: Meningitis Ontology; IDOPlant: Plant Disease Ontology; IDOSCHISTO: Schistosomiasis Ontology; IDOSA; Staphylococcus aureus Infectious Disease Ontology; IAO: Information Artifact Ontology; NARSA: Network on Antimicrobial Resistance in Staphylococcus aureus; NCBITaxon: NCBI organismal classification; OBI: Ontology for Biomedical Investigations; OBO: Open Biomedical Ontologies; OGMS: Ontology for General Medical Science; OWL: Web Ontology Language; RO: Relations Ontology; VIDO: IDO Virus.
\end{abstract}

\title{
Declarations
}

Ethics approval and consent to participate

Not applicable

\section{Consent for publication}

Not applicable

\section{Availability of Data and Materials}

The datasets generated and/or analysed during the current study are freely publicly available in the IDO Core GitHub repository [https://github.com/infectious-disease-ontology/infectious-disease-ontology] as well as online ontology repositories such as Ontobee. [http://www.ontobee.org/ontology/IDO] and BioPortal [http://www.ontobee.org/ontology/IDO]. IDO extensions are also freely publicly available on Github, Ontobee and BioPortal.

\section{Competing interests}

The authors declare that they have no competing interests.

\section{Funding}

BS's contributions were supported by the NIH under NCATS 1UL1TR001412 (Buffalo Clinical and Translational Research Center). SB's and JB's contributions were supported by NIH / NLM T5 Biomedical Informatics and Data Science Research Training Programs (5T15LM012495-03).

\section{Author's contributions}

All authors read and extensively reviewed the manuscript. SB and JB wrote the manuscript and conducted the research. BS and LGC were principal developers of IDO Core. BS is a principal developer of BFO and OGMS. JB, BS and SB are the principal developers of VIDO and IDO-COVID-19. 


\section{Acknowledgements}

We would like to acknowledge the following for their contributions to IDO Core: Alex Diehl, Alan

Ruttenberg, Albert Goldfain, Bjoern Peters, and Jie Zheng. This paper has benefitted from feedback from Alex Diehl, Chris Stoeckert, Oliver He, Asiyah Yu Lin, and Werner Ceusters.

\section{References}

1. Pesquita C, Ferreirra JD, Couto FM, Silva MJ. The epidemiology ontology: an ontology for the semantic annotation of epidemiological resources. J Biomed Semant. 2014; doi:10.1186/ 2041-1480-5-4.

2. Arp R, Smith B, Spear A. Building Ontologies with Basic Formal Ontology. Cambridge, MA: MIT Press; 2015.

3. Zeng ML, Hong Y, Clunis J, He S, Coladangelo LP. Implications of Knowledge Organization Systems for Health Information Exchange and Communication during the COVID-19 Pandemic. Data Information Management. 2020; 4(3). doi:10.2478/dim-2020-0009.

4. The Gene Ontology Consortium. The Gene Ontology Resource: 20 years and still GOing. Nucleic Acids Res. 2019; 47:D330-D338. doi:10.1093/nar/gky1055.

5. Smith B, Ashburner M, Rosse C, Bard J, Bug W, Ceusters W, et al. The OBO Foundry: coordinated evolution of ontologies to support biomedical data integration. Nat Biotechnol. 2007; 25:1251-1255. doi:10.1038/nbt1346.

6. The Open Biomedical Ontologies Foundry. http://obofoundry.org/. Accessed 27 Apr 2020.

7. Smith B, Ceusters W, Klagges B, Köhler J, Kumar A, Lomax J, et al. Relations in biomedical ontologies. Genome Biol. 2005; 6:R46. doi:10.1186/gb-2005-6-5-r46.

8. ISO/IEC 21838-2. https://www.iso.org/standard/74572.html. Accessed 27 Apr 2020.

9. Basic Formal Ontology (BFO) 2020, https://basic-formal-ontology.org/bfo-2020.html. Accessed 2 Mar 2021.

10. Cowell LG, Smith B. Infectious Diseases Ontology. In: Sintchenko V, editor. Infectious Disease Informatics. New York, NY: Springer; 2010. p. 373-95.

11. Goldfain A, Smith B, Cowell LG. Dispositions and the infectious disease ontology. In: Galton A, Mizoguchi R, editors. Formal Ontology in Information Systems: Proceedings of the 6th International Conference (FOIS 2010). Amsterdam: IOS Press; 2010. p. 400-413.

12. Scheuermann RH, Ceusters W, Smith B. Toward an ontological treatment of disease and diagnosis. AMLA Summit on Translat Bioinform. 2009; p. 116-120.

13. Rupnik M, Wilcox MH, Gerding DN. Clostridium difficile infection: New developments in epidemiology and pathogenesis. Nat Rev. Microbiol. 2009. 7(7): 526-36. doi:10.1038/nrmicro2164.

14. Bruchfeld J, Correia-Neves M, Källenius G. Tuberculosis and HIV Coinfection. Cold Spring Harb Perspect Med. 2015; 5(7): a017871. doi:10.1101/cshperspect.a017871.

16. Bandrowski A, Brinkman R, Brochhausen M, Brush MH, Bug B, Chibucos MC, et al. The Ontology for Biomedical Investigations. PLOS ONE. 2016; 11(4):e0154556. doi: 10.1371/journal.pone.0154556.

17. https://github.com/obi-ontology/obi/issues/1306. Accessed 4 Mar 2021.

18. Federhen S. The NCBI Taxonomy Database. Nucleic Acids Res. 2012; 40:D136-D143. doi: 10.1093/nar/gkr1178.

19. McNicholl JM, Smith DK, Qari SH, Hodge T. Host Genes and HIV: The role of the chemokine receptor gene CCR5 and its allele (A32 CCR5). Emerg Infect Dis. 1997; 3(3):261-271. doi:10.3201/eid0303.970302.

20. Pathogen Transmission Ontology. https://bioportal.bioontology.org/ontologies/PTRANS. Accessed 27 Apr 2020.

21. Goldfain A, Smith B, Cowell LG. Towards an ontological representation of resistance: the case of MRSA.J Biomed Inform. 2011; 44:35-41. doi:10.1016/j.jbi.2010.02.008.

22. Hogan WR, Wagner MM, Brochhausen M, Levander J, Brown ST, Millet N. The Apollo Structured Vocabulary: an OWL2 ontology of phenomena in infectious disease epidemiology and population biology for use in epidemic simulation. J Biomed Semant. 2016; 7(50). doi:10.1186/s13326-016-0092-y.

23. Ceusters W, Smith B. About: towards foundations for the information artifact ontology. In: Couto FM, Hasting J, editors. Proceedings of the $6^{\text {th }}$ International Conference on Biomedical Ontology (ICBO 2015). CEUR-WS.org; 2015. P. 1-5.

24. Liu Y, Chan W, Wang Z, Hur J, Xie J, Yu H, et al. Ontological and bioinformatic analysis of anti-coronavirus drugs and their implication for drug repurposing against COVID-19. Preprints. https://doi.org/10.20944/ preprints202003.0413.v1 (2020). Accessed 27 Apr 2020. 
25. Ong E, Wong M, Huffman A, He Y. COVID-19 coronavirus vaccine design using reverse vaccinology and machine learning. bioRxiv. https://doi.org/10.1101/2020.03.20.000141 (2020). Accessed 27 April 2020.

26. He Y, Yu H, Ong E, Wang Y, Liu Y, Huffman A, et al. CIDO, a community-based ontology for coronavirus disease knowledge and data integration, sharing, and analysis. Sci Data. 2020; 7(181). doi:10.1038/s41597-020-05236.

27. Coronavirus Infectious Disease Ontology. https://bioportal.bioontology.org/ontologies/CIDO. Accessed 27 Apr 2020.

28. Luciano J, Schriml L, Squires B, Scheuermann R. The Influenza Infectious Disease Ontology (I-IDO). The 11th Annual Bio-Ontologies Meeting, ISMB. 2008, 20 July; Toronto, Canada.

29. Influenza Ontology. https://bioportal.bioontology.org/ontologies/FLU. Accessed 27 Apr 2020.

30. Lin Y, Xiang Z, He Y. Brucellosis ontology (IDOBRU) as an extension of the infectious disease ontology. J Biomed Semant. 2011; doi:10.1186/2041-1480-2-9.

31. Brucellosis Ontology. https:// bioportal.bioontology.org/ontologies/IDOBRU. Accessed 27 Apr 2020.

32. Beverley J, Smith B, Babcock S, Cowell L. Coordinating coronavirus research: the COVID-19 Infectious Disease Ontology. OSF Preprints. https://osf.io/5bx8c/ (2020). Accessed 20 Sept 2020.

33. Virus Infectious Disease Ontology. https://bioportal.bioontology.org/ontologies/VIDO. Accessed 15 Jun 2020.

34. COVID-19 Infectious Disease Ontology. https://bioportal.bioontology.org/ontologies/IDO-COVID-19. Accessed 15 Jun 2020.

35. Mitraka E, Topalis P, Dritsou V, Dialynas E, Louis C. Describing the breakbone fever: IDODEN, an ontology for dengue fever. PLOS Negl Trop Dis. 2015; 9(2): e0003479. doi:10.1371/journal.pntd.0003479.

36. Dengue Ontology. https://bioportal.bioontology.org/ontologies/IDODEN. Accessed 27 Apr 2020.

37. Topalis P, Mitraka E, Bujila I, Deligianni E, Dialynas E, Siden-Kiamos I, et al. IDOMAL: an ontology for malaria. Malar J. 2010; 9(230). doi:10.1186/1475-2875-9-230.

38. Malaria Ontology. https://github.com/VeuPathDB-ontology/IDOMAL. Accessed 27 Apr 2020.

39. Béré C, Camara G, Malo S, Lo M, Ouaro S. IDOMEN: an extension of infectious disease ontology for MENingitis. In: Ohno-Machado L, Séroussi B, editors. MEDINFO 2019: Health and Wellbeing e-Networks for All. Amsterdam: IOS Press; 2019. P. 313-317.

40. Meningitis Ontology. https://github.com/cedricbere/IDOMEN. Accessed 27 Apr 2020.

41. Walls RL, Smith B, Elser J, Goldfain A, Stevenson DW, Jaiswal P. A plant disease extension of the infectious disease ontology. In: Cornet R, Stevens R, editors. Proceedings of the $3^{\text {rd }}$ International Conference on Biomedical Ontology. CEURSWS.org; 2012. P. 1-5.

42. Plant Disease Ontology. http://purl.obolibrary.org/obo/idoplant.owl. Accessed 27 Apr 2020.

43. Goldfain A, Smith B, Cowell LG. Constructing a lattice of infectious disease ontologies from a staphylococcus aureus isolate repository. In: Cornet R, Stevens R, editors. Proceedings of the $3^{\text {rtd }}$ International Conference on Biomedical Ontology (ICBO 2012). CEURS-WS.org; 2012. P. 1-5.

44. Staphylococcus aureus Infectious Disease Ontology. https://github.com/awqbi/ido-staph. Accessed 27 Apr 2020.

45. Camara G, Desprès S, Lo M. IDOSCHISTO: une extension de l'ontologie noyau des maladies infectieuses (IDOCore) pour la schistosomiases. In: Faron-Zucker C, editor. IC - 25èmes Journées francophones d'Ingénierie des Connaissances, Clermont-Ferrand, France. Session 1: Construction, peuplement et exploitation d'ontologies. 2014. P. 39-50.

46. Schistosomiasis Ontology. https://github.com/gaoussoucamara/idoschisto. Accessed 27 Apr 2020.

47. Sargeant D, Deverasetty S, Strong CL, Alaniz IJ, Bartlett A, Brandon NR, et al. The HIVToolbox 2 Web System Integrates Sequence, Structure, Function and Mutation Analysis. PLOS ONE. 2014; 9:e98810. doi: 10.1371/journal.pone.0098810.

48. HIV Ontology. https:// bioportal.bioontology.org/ontologies/HIV. Accessed 27 Apr 2020.

49. Network on Antimicrobial Resistance in Staphylococcus aureus. http://www.narsa.net/. Accessed 27 Apr 2020.

50. Kuhn J. Virus Taxonomy. Reference Modules in Life Sciences. 2020; doi:10.1016/B978-0-12-809633-8.21231-4.

51. Xiang Z, Courtot M, Brinkman RR, Ruttenberg A, He Y. OntoFox: webbased support for ontology reuse. BMC research notes. 2010; 3:175. doi:10.1186/1756-0500-3-175.

52. Schober D, Smith B, Lewis SE, Kusnierczyk W, Lomax J, Mungall C, et al. Survey-based naming conventions for use in OBO Foundry ontology development. BMC Bioinformatics. 2009; 10(125). doi:10.1186/1471-2105-10-125.

53. Baltimore D. Expression of Animal Virus Genomes. Bacteriological Reviews. 1971; 35(3). P. 235-41. 
54. Symptom Ontology. https://bioportal.bioontology.org/ontologies/SYMP. Accessed 3 Aug 2020.

55. Coronavirus Disease 2019 (COVID-19) 2020 Interim Case Definition, Approved April 2, 2020. Centers for Disease Control and Prevention. 2020; https://wwwn.cdc.gov/nndss/conditions/coronavirus-disease-2019-covid-19/casedefinition/2020/. Accessed 3 Aug 2020.

56. Standardization Surveillance Case Definition and National Notification for 2019 Coronavirus Disease (COVID-19). Council of State of Territorial Epidemiologists. 2020; https://asprtracie.hhs.gov/technicalresources/resource/8322/standardized-surveillance-case-definition-and-national-notification-for-2019-novelcoronavirus-disease-covid-19. Accessed 3 Aug 2020.

57. Sayers S, Li L, Ong E, Deng S, Fu G, Lin Y, et al. Victors: a web-based knowledge base of virulence factors in human and animal pathogens. Nucleic Acids Res. 2019; 47:D693-D700. doi:10.1093/nar/gky999.

58. Zhou Y, Hou Y, Shen J, Huang Y, Martin W, Cheng F. Network-based Drug Repurposing for Novel Coronavirus 2019-nCoV/SARS-CoV-2. Cell Discovery. 2020; 6(14). doi:10.1038/s41421-020-0153-3.

59. Degtyarenko K, Matos P, Ennis M, Hastings J, Zbinden M, Mcnaught A, et al. ChEBI: a database and ontology for chemical entities of biological interest. Nucleic Acids Res. 2008; 36:D344-D350. doi:10.1093/nar/gkm791.

60. Haendel MA, Balhoff JP, Bastian FB, Blackburn DC, Blake JA, Bradford Y, et al. Unification of multi-species vertebrate anatomy ontologies for comparative biology in Uberon. J Biomed Semant. 2014; doi:10.1186/2041-1480-521.

61. He Y, Cowell LG, Diehl AD, Mobley H, Peters B, Ruttenberg A, et al. VO: Vaccine Ontology. In: Smith B, editor. Proceedings of the $1^{1: t}$ International Conference on Biomedical Ontology (ICBO 2009). Buffalo: NCOR; 2009. P. 172.69.

62. Li F. Structure, Function, and Evolution of Coronavirus Spike Proteins. Annu Rev Virol. 2016; 3(1): 237-261. doi:10.1146/annurev-virology-110615-042301.

63. Schoeman D, Fielding BC. Coronavirus envelope protein: current knowledge. Virol J. 2019; 16(69). doi: 10.1186/s12985-019-1182-0.

64. Letko M, Marzi A, Munster V. Functional assessment of cell entry and receptor usage for SARS-CoV-2 and other lineage B betacoronaviruses. Nat Microbiol. 2020; 5:562-569. doi:10.1038/s41564-020-0688-y.

65. Chen Y, Liu Q, Guo D. Emerging coronaviruses: Genome structure, replication, and pathogenesis. J Med Virol. 2020; 92: 418-423. doi:10.1002/jmv.2568.

66. Natale DA, Arighi CN, Blake JA, Bona J, Chen C, Chen S, et al. Protein Ontology (PRO): enhancing and scaling up the representation of protein entities. Nucleic Acids Res. 2017; 45: D339-D346. doi: 10.1093/nar/gkw1075.

67. Hoffmann M, Kleine-Weber H, Schroeder S, Krüger N, Herrler T, Erichsen S, et al. SARS-CoV-2 Cell Entry Depends on ACE2 and TMPRSS2 and Is Blocked by a Clinically Proven Protease Inhibitor. Cell. 2020; 181(2): 271280 e278. doi:10.1016/j.cell.2020.02.052.

68. WHO COVID-19 Rapid Version CRF. https://bioportal.bioontology.org/ ontologies/COVIDCRFRAPID. Accessed 27 Apr 2020.

69. COVID-19 Surveillance Ontology. https://bioportal.bioontology.org/ontologies/COVID19. Accessed 27 Apr 2020.

70. Linked COVID-19 Data Ontology. https://github.com/Research-Squirrel-Engineers/COVID-19. Accessed 27 Apr 2020.

71. COVID-19 Research Knowledge Graph. https://github.com/nasa-jpl-cord-19/covid19-knowledge-graph. Accessed 27 Apr 2020.

72. Scheuermann R, Kong M, Dahlke C, Cai J, Lee J, Qian Y, et al. Ontology-based knowledge representation of experiment metadata in biological data mining. In: Chen J, Lonardi S, editors. Biological Data Mining. Boca Raton, FL: Chapman \& Hall; 2009. P. 529-559.

73. Schriml L, Chuvochina M, Davies N, Eloe-Fadrosh, E, Finn R, Hugenholtz P, et al. COVID-19 pandemic reveals the peril of ignoring metadata standards. Sci Data. 2020; 7(188). doi:10.1038/s41597-020-0524-5.

74. National Library of Medicine. https://www.nlm.nih.gov/. Accessed 20 Sept 2020.

75. Liu-Wei W, Kafkas Ş, Chen J, Tegnér J, Hoehndorf R. Prediction of novel virus-host interactions by integrating clinical symptoms and protein sequences. bioRxiv. https://doi.org/10.1101/2020.04.22.055095 (2020). Accessed 27 Apr 2020.

76. Huntley RP, Sawford T, Mutowo-Meullenet P, Shypitsyna A, Bonilla C, Martin MJ, et al. The GOA database: Gene Ontology annotation updates for 2015. Nucleic Acids Res. 2015; 43: D1057-D1063. doi: 10.1093/nar/gku1113 
77. Squires RB, Noronha J, Hunt V, García-Sastre A, Macken C, Baumgarth N, et al. Influenza Research Database: An integrated bioinformatics resource for influenza virus research. Influenza Other Respir Viruses. 2012; 6(6), 404-416. doi:10.1111/j.1750-2659.2011.00331.x.

78. Kulmanov M, Smaili FZ, Gao X, Hoehndorf R, Machine learning with biomedical ontologies. bioRxiv. https://doi.org/10.1101/2020.05.07.082164 (2020). Accessed 15 Jul 2020.

79. BFO 2.0 Users Guide. http://purl.obolibrary.org/obo/bfo/Reference. Accessed 17 Apr 2021.

80. https://www.youtube.com/channel/UC8rDbmRGP6A2bs6tn0AOErQ. Accessed 17 Apr 2021.

81. BFO Discussion Group. https://groups.google.com/g/bfo-discuss. Accessed 17 Apr 2021.

82. https://github.com/OBOFoundry/OBOFoundry.github.io/issues. Accessed 17 Apr 2021.

83. https://obo-communitygroup.slack.com/?redir=\%2Farchives\%2FC01DP18L5GW. Accessed 17 Apr 2021.

84. https://github.com/infectious-disease-ontology-extensions. Accessed 20 Feb 2020.

85. Protégé. http://protege.stanford.edu. Accessed 27 Apr 2020.

86. https://github.com/infectious-disease-ontology/infectious-disease-ontology. Accessed 27 Apr 2020.

\section{Additional Files}

Additional File 1: Supplementary Tables and Related Discussion (.docx)

Table S1 | Ontologies building on the OGMS treatment of disease and diagnosis; Table S2 | Overview of IDO extension ontologies that have been developed or planned; Table S3 | Some other ontologies within the infectious disease domain that make use of IDO Core. Table S4 | IDOBRU Hierarchy; Table S5 | Some databases to which IDO annotations have been applied; Table S6 | IDO based Decision Support Systems

Additional File 2: The Infectious Disease Ontology Extensions: Some Issues. (.docx) Several IDO ontologies require significant reengineering if they are to be considered bona fide extensions of IDO Core. This document provides an overview of some issues concerning specific IDO extensions, while providing some suggestions for how they can be addressed.

Additional File 3: Case Study: IDOSA and methicillin resistant Staphylococcus aureus (.docx)

\section{Additional File 1: Supplementary Tables and Related Discussion}

The Ontology for General Medical Science (OGMS) covers types of entities relevant to clinical encounters between doctor and patient. Thus, it includes representations of disease, causes and manifestations of disease, diagnosis, symptom, treatment, patient examination, history taking, laboratory test, and so forth.

Table S1 | Ontologies building on the OGMS treatment of disease and diagnosis

\begin{tabular}{|l|l|}
\hline Agronomy Ontology (Agro) [1] & Apollo Structured Vocabulary (Apollo-SV) [2] \\
\hline Beta Cell Genomics Ontology (BCGO) [3] & Cardiovascular Disease Ontology (CVDO) [4] \\
\hline Coronavirus Infectious Disease Ontology (CIDO) [5] & Human Disease Ontology (DOID) [6] \\
\hline Drug Ontology (DrugON) [7] & VEuPathDB ontology (EUPATH) [8] \\
\hline Influenza Ontology (IDOFLU) [9] & Genomic Epidemiology Ontology (GenEpio) [10] \\
\hline
\end{tabular}




\begin{tabular}{|l|l|}
\hline Hypertension Ontology (HTN) [11] & Infectious Disease Ontology (IDO) [12] \\
\hline Brucellosis Ontology (IDOBRU) [13] & Meningitis Ontology (IDOMEN) [14] \\
\hline Plant Disease Ontology (IDOPlant) [15] & $\begin{array}{l}\text { Staph aureus Infectious Disease Ontology (IDOSA) } \\
{[16]}\end{array}$ \\
\hline Schistosomiasis Ontology (IDOSCHISTO) [17] & Mental Functioning Ontology (MFO) [18] \\
\hline Mental Diseases Ontology (MFOMD) [19] & Monarch Disease Ontology (MonDO) [20] \\
\hline Neurological Disease Ontology (ND) [21] & Ontology of Adverse Events (OAE) [22] \\
\hline \begin{tabular}{l} 
Ontology for Biomedical Investigations (OBI) [23] \\
\hline Ontology for Biobanking (OBIB) [25]
\end{tabular} & OBI-NIAID-GSC-BRC-view [24] \\
\hline $\begin{array}{l}\text { Oral Health and Disease Ontology (OHD) [27] } \\
\text { Ontology of Laboratory Animal Medicine (OLAM) } \\
{[29]}\end{array}$ & Ontology of Host Pathogen Interactions (OHPI) [28] \\
\hline $\begin{array}{l}\text { Ontology of Medically Relevant Social Entities } \\
\text { (OMRSE) [31] }\end{array}$ & Obstetric and Neonatal Ontology (ONTONEO) [32] \\
\hline $\begin{array}{l}\text { Ontology of Precision Medicine and Investigation } \\
\text { (OPMI) [33] }\end{array}$ & Prescription of Drugs Ontology (PDRO) [34] \\
\hline \begin{tabular}{l} 
Planarian Phenotype Ontology (PLANP) [35] \\
\hline \begin{tabular}{l} 
Vital Sign Ontology (VSO) [37] \\
\hline
\end{tabular}
\end{tabular} & Vaccine Ontology (VO) [36] \\
\hline
\end{tabular}

Table S2 | Overview of IDO extension ontologies that have been developed or planned

\begin{tabular}{|l|l|}
\hline $\begin{array}{l}\text { Malaria } \\
\text { Ontology } \\
\text { (IDOMAL) [38] }\end{array}$ & $\begin{array}{l}\text { Created by the Christos Louis team at the Institute of Molecular Biology and } \\
\text { Biochemistry (IMBB) in Crete, Greece, IDOMAL covers both clinical and } \\
\text { epidemiological aspects of malaria, disease and vector biology, as well as } \\
\text { intervention attempts to control the disease. IDOMAL was originally } \\
\text { developed in the context of VectorBase. }\end{array}$ \\
\hline $\begin{array}{l}\text { Dengue } \\
\text { Ontology } \\
\text { (IDODEN) [39] }\end{array}$ & $\begin{array}{l}\text { Also developed by the Louis research team at IMBB, IDODEN covers all } \\
\text { aspects of dengue fever including disease biology, epidemiology, clinical } \\
\text { features, and vector entomology. IDODEN was developed in the context of } \\
\text { VectorBase and its structure was designed to mirror that of IDOMAL. } \\
\text { Intended for use in Dengue decision support systems. }\end{array}$ \\
\hline $\begin{array}{l}\text { Brucellosis } \\
\text { (IDOBdogy }\end{array}$ & $\begin{array}{l}\text { Developed by Yongqun He and his team at the University of Michigan, } \\
\text { IDOBRU focuses on Brucellosis, a highly contagious zoonotic infectious } \\
\text { disease caused by the intracellular, Gram-negative bacteria Brucella. IDOBRU } \\
\text { encompasses the domains of clinical care, public health, and biomedical } \\
\text { research, along seven major axes: host infection and zoonotic disease } \\
\text { transmission, symptoms, virulence factors and pathogenesis, diagnosis, } \\
\text { intentional release, vaccine prevention, and treatment. }\end{array}$ \\
\hline $\begin{array}{l}\text { Influenza } \\
\text { Ontology } \\
\text { (IDOFLU) [9] }\end{array}$ & $\begin{array}{l}\text { Developed by Richard Scheuermann, Lynn Schriml, Joane Luciano, and } \\
\text { Burke Squires, IDOFLU covers the natural, experimental and clinical realms } \\
\text { related to influenza virus life cycle, infection and disease. IDOFLU utilizes }\end{array}$ \\
\hline
\end{tabular}




\begin{tabular}{|c|c|}
\hline & $\begin{array}{l}\text { OBI classes for components of materials, qualities, and processes to map } \\
\text { influenza virus sequence and surveillance terms to their corresponding } \\
\text { materials and qualities. IDOFLU is applied to data collected by the Centers } \\
\text { for Excellence in Influenza Research and Surveillance (CEIRS) project to } \\
\text { help researchers more easily elucidate influenza virulence and pathogenesis } \\
\text { etiology. }\end{array}$ \\
\hline $\begin{array}{l}\text { Staphylococcus } \\
\text { aureus } \\
\text { Infectious } \\
\text { Disease } \\
\text { Ontology } \\
\text { (IDOSA) [16] }\end{array}$ & $\begin{array}{l}\text { Developed by Lindsay Cowell, Barry Smith and Albert Goldfain, in } \\
\text { collaboration with Dr. Vance Fowler at Duke University Medical Center, } \\
\text { IDOSA focuses on Staph aureus (Sa) infection diseases. The ontology is } \\
\text { used to analyze networks of functionally related gene products to identify } \\
\text { host genes conferring susceptibility to Staphylococcus aureus bacteremia and } \\
\text { has the potential to provide a classification of Sa that allows automated } \\
\text { inference of resistance profiles. }\end{array}$ \\
\hline $\begin{array}{l}\text { HIV Ontology } \\
\text { (IDOHIV) }\end{array}$ & $\begin{array}{l}\text { Developed by Martin Schiller of UNLV, the HIV ontology is intended to } \\
\text { cover all types of HIV data and information; intended for use in the } \\
\text { HIVToolbox web application [40]. }\end{array}$ \\
\hline $\begin{array}{l}\text { Schistosomiasis } \\
\text { Ontology } \\
\text { (IDOSCHISTO) } \\
\text { [17] }\end{array}$ & $\begin{array}{l}\text { Developed by team of researchers based in Senegal led by Dr. Gaoussou } \\
\text { Camara, IDOSCHISTO focuses on schistosomiasis, a waterborne infectious } \\
\text { disease caused by Schistosoma helminth parasites. The ontology is organized } \\
\text { into } 3 \text { main sub-modules; i) Biology, e.g. pathogen/host interactions, host } \\
\text { physiological reactions to the disease, pathogen taxonomy and life-cycle; ii) } \\
\text { Epidemiology, for example risk factors, spread of disease, means to prevent } \\
\text { and control; and iii) Clinical, including symptoms that influence differential } \\
\text { diagnoses and treatment decisions. }\end{array}$ \\
\hline $\begin{array}{l}\text { Plant Disease } \\
\text { Ontology } \\
\text { (IDOPlant) [15] }\end{array}$ & $\begin{array}{l}\text { Under active development within the context of the Planteome Project, } \\
\text { IDOPlant provides a comprehensive reference ontology for any infectious } \\
\text { plant disease. The main aims of IDOPlant are to "provide plant scientists } \\
\text { with the means identify genomic and genetic signatures host-pathogen } \\
\text { interactions, resistance, or susceptibility, and to help agronomists and } \\
\text { farmers by developing tools to identify disease phenotypes and gather } \\
\text { epidemiological statistics" [41]. }\end{array}$ \\
\hline $\begin{array}{l}\text { Meningitis } \\
\text { Ontology } \\
\text { (IDOMEN) [14] }\end{array}$ & $\begin{array}{l}\text { Also developed by Dr. Camara's team, IDOMEN focuses on meningitis, a } \\
\text { disease caused by the gram-negative bacteria, Neisseria meningitidis. IDOMEN } \\
\text { covers the meningitis domain along three main axes: i) biological (immunity, } \\
\text { virulence factors, pathogen and host biology); ii) clinical (clinical } \\
\text { manifestations, laboratory tests and findings, diagnosis and treatment); iii) } \\
\text { epidemiological (surveillance, prevention, epidemic emergence factors such } \\
\text { as risk behaviors, climate and environment). IDOMEN is designed to assist } \\
\text { in the analysis and filtering of data collected on social media platforms such } \\
\text { as Twitter to help improve the early detection of meningitis epidemic risks in } \\
\text { sub-Saharan Africa. Increasingly, search queries, social media posts, and web } \\
\text { server access logs have been employed for disease surveillance. Digital } \\
\text { platforms provide real time data streams from which information related to } \\
\text { public health can be extracted in a timely manner at low to virtually no cost. } \\
\text { By mining these data sources for traces of health-related activities, they can } \\
\text { be transformed into useful metrics for inclusion in statistical estimation } \\
\text { models for disease incidence. Google, Wikipedia and Twitter have each been }\end{array}$ \\
\hline
\end{tabular}




\begin{tabular}{|c|c|}
\hline & $\begin{array}{l}\text { investigated as tools for quantifying disease incidence rates [42-44]. } \\
\text { Ontology-based approaches to internet-based disease surveillance have also } \\
\text { been developed and applied with good success }[45,46] \text {. }\end{array}$ \\
\hline $\begin{array}{l}\text { Coronavirus } \\
\text { Infectious } \\
\text { Disease } \\
\text { Ontology } \\
\text { (CIDO) [5] }\end{array}$ & $\begin{array}{l}\text { Initiated by Yongqun He and Hong Yu, CIDO was developed by a } \\
\text { collaborative group of researchers in both the US and in China in response } \\
\text { to the recent COVID-19 outbreaks. CIDO provides a standardized human- } \\
\text { and computer-interpretable annotation and representation of various } \\
\text { coronavirus infectious diseases including their etiology, transmission, } \\
\text { epidemiology, pathogenesis, diagnosis, prevention and treatment. }\end{array}$ \\
\hline $\begin{array}{l}\text { IDO } \\
\text { Tuberculosis } \\
\text { (IDOTUB) }\end{array}$ & Planned, but is not yet currently in development. \\
\hline $\begin{array}{l}\text { IDO Infective } \\
\text { Endocarditis }\end{array}$ & $\begin{array}{l}\text { Planned, but not yet currently in development. Preliminary work is available } \\
\text { online: https:// sivsmusings. wordpress.com/ }\end{array}$ \\
\hline
\end{tabular}

Table S3 | Some other ontologies within the infectious disease domain that make use of IDO Core

\begin{tabular}{|c|c|}
\hline $\begin{array}{l}\text { Vaccine } \\
\text { Ontology (VO) } \\
{[36]}\end{array}$ & $\begin{array}{l}\text { Developed in collaboration with the IDO Core team, VO has strong ties to } \\
\text { the IDO initiative. Maintained by the He team at the University of Michigan, } \\
\text { VO focuses on the classification of vaccines and vaccine components, } \\
\text { vaccine quality and phenotypes, and host immune response to vaccines. VO } \\
\text { is used in the Vaccine Investigation and Online Information Network } \\
\text { (VIOLIN, http://www.violinet.org/) a central repository for literature related } \\
\text { to, and data resulting from, vaccine research. }\end{array}$ \\
\hline $\begin{array}{l}\text { Bacterial } \\
\text { Clinical } \\
\text { Infectious } \\
\text { Disease } \\
\text { Ontology } \\
\text { (BCIDO) [47] }\end{array}$ & $\begin{array}{l}\text { BCIDO provides a controlled terminology for clinical bacterial infectious } \\
\text { diseases along with domain knowledge commonly used in the hospital in- } \\
\text { patient setting. BCIDO was designed to augment the use of ADSSs, thus } \\
\text { serving as a tool to guide differential bacterial diagnoses and to assist in the } \\
\text { prescribing of appropriate antimicrobial treatments. As such, BCIDO } \\
\text { "encompasses terms and knowledge about common clinical presentations of } \\
\text { [bacterial] infections, patient specific factors that influence differential } \\
\text { diagnoses and treatment options, the [bacteria] themselves, and the } \\
\text { antimicrobial agents used to treat infections" [47]. Though not an IDO } \\
\text { extension per se, BCIDO imports IDO Core in full. }\end{array}$ \\
\hline $\begin{array}{l}\text { Vector } \\
\text { Surveillance and } \\
\text { Management } \\
\text { Ontology } \\
\text { (NSMO) [48] }\end{array}$ & $\begin{array}{l}\text { Developed by a team of researchers at Colorado State University led by Drs. } \\
\text { Lars Eisen and Saul Lozano, in collaboration with Dr. Cowell, VSMO covers } \\
\text { the domain of surveillance and management of vectors and vector-borne } \\
\text { pathogens, with special emphasis on content to support operational activities } \\
\text { through inclusion in databases, data management systems and decision } \\
\text { support systems [48]. The ontology includes terms for i) arthropod species } \\
\text { capable of being biological vectors and for pathogen species transmitted by } \\
\text { arthropod vectors; ii) chemical compounds relevant to insecticide resistance } \\
\text { and chemical pesticide active substances (originating from MIRO); iii) terms } \\
\text { for equipment used to collect or control vectors or vertebrae pathogen hosts, } \\
\text { and tools used to kill vectors or prevent contact with humans. In consultation } \\
\text { with the IDO Core developers, the relation bas_vector was created for VSMO } \\
\text { to link pathogens to their biological vectors. }\end{array}$ \\
\hline
\end{tabular}




\begin{tabular}{|l|l|}
\hline $\begin{array}{l}\text { Genomic } \\
\text { Epidemiology } \\
\text { Ontology } \\
\text { (GenEpio) [10] }\end{array}$ & $\begin{array}{l}\text { GenEpio is a controlled vocabulary for infectious disease surveillance and } \\
\text { outbreak investigations implementing whole genome sequencing (WGS) of } \\
\text { microbial pathogens. GenEpio aims to enable the integration and promotion } \\
\text { of all contextual information required to interpret pathogen genomics data, } \\
\text { including critical knowledge about sequencing pipelines and sequence quality; } \\
\text { lab results describing antimicrobial resistance and virulence phenotypes; } \\
\text { epidemiological data concerning potential sources of risk and exposure; as } \\
\text { well as data about susceptible populations and geographical distributions of } \\
\text { pathogen strain [10]. GenEpio is currently being integrated into IRIDA } \\
\text { (Integrated Rapid Infectious Disease Analysis), a user-friendly, decentralized, } \\
\text { open-source bioinformatics and analytical web platform to support real-time } \\
\text { infectious disease outbreak investigations using WGS data [49]. }\end{array}$ \\
\hline
\end{tabular}

\section{Examples: IDOBRU \& IDOPlant}

Two ontologies from Table S2 provide examples of excellent ontology design. IDOBRU, the Brucellosis Infectious Disease Ontology, is maintained by the He research team at the University of Michigan, and is used to facilitate the integration and exchange of brucellosis information stored in widely used databases, including:

The Brucella Bioinformatics Portal [50]: a portal for the search and analysis of individual Brucella genes; linked to more than 20 other databases and programs.

The Vaccine Investigation and Online Information Network (VIOLIN) [51] a central repository for literature related to, and data resulting from, vaccine research.

IDOBRU exhibits a well-organized hierarchy with BFO, OGMS, and IDO Core imported in full, and is a good exemplar of the IDO Core hub and spokes model, as illustrated in Table S4.

Table S4 | IDOBRU Hierarchy

\begin{tabular}{|c|c|c|}
\hline IDOBRU Axis & Top Level IDOBRU Classes & $\begin{array}{l}\text { Imported OBO Ontology Class } \\
\text { from which it descends }\end{array}$ \\
\hline $\begin{array}{l}\text { host infection and } \\
\text { zoonotic disease } \\
\text { transmission }\end{array}$ & $\begin{array}{l}\text { process of establishing Brucella infection } \\
\text { in host, } \\
\text { Brucella infectious disposition, } \\
\text { Brucella host role }\end{array}$ & $\begin{array}{l}\text { process of establishing an infection (IDO } \\
\text { Core) } \\
\text { zoonotic disposition (IDO Core) } \\
\text { infectious agent host role (IDO Core) }\end{array}$ \\
\hline $\begin{array}{l}\text { virulence factors } \\
\text { and pathogenesis }\end{array}$ & $\begin{array}{l}\text { Brucella virulence factor, } \\
\text { Brucella virulence factor disposition }\end{array}$ & $\begin{array}{l}\text { virulence factor (IDO Core), } \\
\text { virulence factor disposition (IDO Core), }\end{array}$ \\
\hline symptoms & brucellosis symptom & symptom (OGMS) \\
\hline diagnosis & brucellosis diagnosis & diagnosis (OGMS) \\
\hline intentional release & Brucella intentional release & planned process $(\mathrm{OBI})$ \\
\hline vaccine prevention & brucellosis vaccine & vaccine (VO) \\
\hline treatment & brucellosis treatment & treatment (OGMS) \\
\hline
\end{tabular}

The other example worth noting is IDOPlant, [15] a plant infectious disease ontology being developed under the auspices of the Planteome Project, which maintains a large database of annotations from plant genomic and phenomic studies [52]. IDOPlant leverages IDO Core in axioms such as the following: 
IDOPlant:process of establishing a Xanthomonas oryzae infection subclass-of IDOPlant:process of establishing a plant bacterial infection,

IDOPlant:process of establishing a plant bacterial infection subclass-of IDO:process of establishing an infection

IDOPlant:rice bacterial leaf blight disease subclass-of IDOPlant:plant bacterial disease

IDOPlant:plant bacterial disease subclass-of IDOPlant:plant infectious disease

IDOPlant:plant infectious disease subclass-of IDO:infectious disease

Table S5 | Some databases to which IDO annotations have been applied

\begin{tabular}{|c|c|}
\hline $\begin{array}{l}\text { The Eukaryotic } \\
\text { Pathogen Genomics } \\
\text { Database } \\
\text { (EuPathDB) [53] }\end{array}$ & $\begin{array}{l}\text { Provides genomic and other data for eukaryotic pathogens including } \\
\text { Cryptosporidium, Giardia, Plasmodium, Theileria, Toxoplasma, and Trichomonas } \\
\text { strains. Maintained by a team of researchers at the University of } \\
\text { Pennsylvania led by Chris Stoeckert. }\end{array}$ \\
\hline VectorBase [54] & $\begin{array}{l}\text { Provides genomic and other data for a variety of invertabrae vectors of } \\
\text { human pathogens. Also maintained by the Stoeckert team. }\end{array}$ \\
\hline $\begin{array}{l}\text { Eukaryotic } \\
\text { Pathogen, Host \& } \\
\text { Vector Genomics } \\
\text { Resource } \\
\text { (VeuPathDB) }\end{array}$ & $\begin{array}{l}\text { With support from a recently awarded 5-year contract with the National } \\
\text { Institute of Allergy and Infectious Diseases, worth up to } \$ 7.2 \text { million in } \\
2019-2020 \text { [55], the Stoeckert team has integrated EuPathDB and } \\
\text { VectorBase into one bioinformatics resource, VeuPathDB. IDO Core is } \\
\text { playing a role in this project, as the VeuPathDB application ontology } \\
\text { [56] imports several IDO Core terms such as: human pathogenicity } \\
\text { disposition, infection, infection prevalence, and primary infection, each of which } \\
\text { are used in the annotation of VeuPathDB datasets. }\end{array}$ \\
\hline $\begin{array}{l}\text { Influenza Research } \\
\text { Database [57] }\end{array}$ & $\begin{array}{l}\text { Resource to elucidate host-influenza virus interactions, leading to new } \\
\text { treatments and preventive action. Contains "surveillance data, human } \\
\text { clinical data associated with virus extracts, phenotypic characteristics of } \\
\text { viruses isolated from extracts, and all genomic and proteomic data } \\
\text { available in public repositories for influenza viruses" [58]. }\end{array}$ \\
\hline $\begin{array}{l}\text { Virus Pathogen } \\
\text { Resource [59] }\end{array}$ & $\begin{array}{l}\text { Database and analysis resource for human pathogenic viruses, including } \\
\text { sequence, surveillance and host response data. }\end{array}$ \\
\hline PHIDIAS [60] & $\begin{array}{l}\text { PHIDIAS (Pathogen-Host Interaction Data Integration and Analysis } \\
\text { System) is a web-based database system for searching, comparing, and } \\
\text { analyzing integrated genome sequences, conserved domains, and gene } \\
\text { expression data related to pathogen-host interactions [60]. }\end{array}$ \\
\hline $\begin{array}{l}\text { Victors virulence } \\
\text { factors database }[60]\end{array}$ & $\begin{array}{l}\text { A database to store and analyze virulence factors of a variety of } \\
\text { pathogens that infect both humans and animals. }\end{array}$ \\
\hline
\end{tabular}

Reflecting their support of knowledge re-use and automated reasoning, ontologies have been implemented in a variety of applications for the enhancement of patient diagnosis, care management and clinical decision support [61-63]. A brief overview and further references are provided in [64]. In the fields of infectious disease, DSSs are commonly used in diagnostic assistance, guidance in the prescription of anti-infectives, biosurveillance, and vector control.

Some examples are provided in Table S6.

Table S6 | IDO based DSSs 


\begin{tabular}{|l|l|}
\hline $\begin{array}{l}\text { Antibiotic decision } \\
\text { support systems } \\
\text { (ADSSs) }\end{array}$ & $\begin{array}{l}\text { Use of ADSSs has been shown to be effective in mitigating } \\
\text { inappropriate antibiotic prescribing and lowering local antimicrobial } \\
\text { resistance [65, 66]. To facilitate interoperability and widespread } \\
\text { circulation of future ADSSs, a Bacterial Clinical Infectious Disease } \\
\text { Ontology (BCIDO) has been developed from IDO Core [47]. }\end{array}$ \\
\hline IDDAP [68] & $\begin{array}{l}\text { IDDAP is a recently developed ontology-driven clinical decision } \\
\text { support system for infectious disease diagnosis and antibiotic } \\
\text { prescription. IDDAP makes use of an infectious disease diagnosis } \\
\text { ontology that builds upon IDO Core. }\end{array}$ \\
\hline $\begin{array}{l}\text { Dengue Decision } \\
\text { Support System } \\
\text { (DDSS) [69, 70] }\end{array}$ & $\begin{array}{l}\text { The DDSS is an ontology driven computational application developed } \\
\text { at Colorado State University to guide the implementation of locally } \\
\text { appropriate Dengue and Dengue Vector control programs. The DDSS is } \\
\text { used in conjunction with Chaak, a cell phone-based system for i) the field capture } \\
\text { of data relating to Dengue vector surveillance; and ii) the rapid transfer } \\
\text { of the data to the central DDSS database [71]. }\end{array}$ \\
\hline
\end{tabular}

\section{References}

1. Devare M, Aubert C, Laporte MA, Valette L, Arnaud E, Buttigieg PL. Data-driven agricultural research for development - a need for data harmonization via semantics. In: Jaiswal P, Hoehndorf R, editors. Proceedings of the Joint International Conference on Biological Ontology and BioCreative (ICBO-BioCreative 2016). CEUR-WS.org; 2016. p. 33.

2. Hogan WR, Wagner MM, Brochhausen M, Levander J, Brown ST, Millet N. The Apollo Structured Vocabulary: an OWL2 ontology of phenomena in infectious disease epidemiology and population biology for use in epidemic simulation. J Biomed Semant. 2016; 7(50). doi:10.1186/s13326-016-0092-y.

3. Zheng J, Manduchi E, Stoeckert C. Development of an Application Ontology for Beta Cell Genomics Based on the Ontology for Biomedical Investigations. In: Dumontier M, Hoehndorf R, Baker CJO, editors. Proceedings of the $4^{\text {th }}$ International Conference on Biomedical Ontology (ICBO 2013). CEUR-WS.org; 2013. p. 62-67.

4. Barton A, Rosier A, Burgun A, Ethier JF. The Cardiovascular disease ontology. In: Garbacz P, Kutz O, editors. Formal Ontology in Information Systems: Proceedings of the 8th International Conference (FOIS 2014). Amsterdam: IOS Press; 2014. p. 409-414.

5. Coronavirus Infectious Disease Ontology. https://bioportal.bioontology.org/ontologies/CIDO. Accessed 27 Apr 2020.

6. Schriml LM, Arze C, Nadendla S, Chang YW, Mazaitis M, Felix V, et al. Disease ontology: a backbone for disease semantic integration. Nucleic Acids Res. 2012; 40:D940-D946. doi: 10.1093/nar/gkr972.

7. Hogan WR, Hanna J, Joseph E, Brochhausen M. Towards a Consistent and Scientifically Accurate Drug Ontology. In: Dumontier M, Hoehndorf R, Baker CJO, editors. Proceedings of the $4^{\text {th }}$ International Conference on Biomedical Ontology (ICBO 2013). CEUR-WS.org; 2013. p. 68-73.

8. Zheng J, Cade JS, Brunk B, Roos DS, Stoeckert CJ, Sullivan SA, et al. Malaria study data integration and information retrieval based on OBO Foundry ontologies. In: Jaiswal P, Hoehndorf R, editors. Proceedings of the Joint International Conference on Biological Ontology and BioCreative (ICBO BioCreative 2016). CEUR-WS.org; 2016. p. 38.

9. Influenza Ontology. https://bioportal.bioontology.org/ontologies/FLU. Accessed 27 Apr 2020.

10. Griffiths E, Dooley D, Graham M, Van Domselaar G, Brinkman FSL, Hsiao WWL. Context Is Everything: Harmonization of Critical Food Microbiology Descriptors and Metadata for Improved Food Safety and Surveillance. Front Microbiol. 2017; 8:1068. doi:10.3389/fmicb.2017.01068.

11. Hypertension Ontology. http://bioportal.bioontology.org/ontologies/HTN. Accessed 27 Apr 2020. 
12. Cowell LG, Smith B. Infectious Diseases Ontology. In: Sintchenko V, editor. Infectious Disease Informatics. New York, NY: Springer; 2010. p. 373-95.

13. Brucellosis Ontology. https:// bioportal.bioontology.org/ontologies/IDOBRU. Accessed 27 Apr 2020.

14. Meningitis Ontology. https://github.com/cedricbere/IDOMEN. Accessed 27 Apr 2020.

15. Plant Disease Ontology. http://purl.obolibrary.org/obo/idoplant.owl. Accessed 27 Apr 2020.

16. Staphylococcus aureus Infectious Disease Ontology. https://github.com/awqbi/ido-staph. Accessed 27 Apr 2020.

17. Schistosomiasis Ontology. https://github.com/gaoussoucamara/idoschisto. Accessed 27 Apr 2020.

18. Hastings J, Ceusters W, Jensen M, Mulligan K, Smith B. Representing mental functioning: Ontologies for mental health and disease. 3rd International Conference on Biomedical Ontology (ICBO 2012). Citeseer; 2012. p. 15.

19. Ceusters W, Smith B. Foundations for a realist ontology of mental disease. J Biomed Semant. 2010; 1(10). doi:10.1186/2041-1480-1-10.

20. Mungall CJ, McMurry JA, Köhler S, Balhoff JP, Borromeo C, Brush M, et al. The Monarch Initiative: an integrative data and analytic platform connecting phenotypes to genotypes across species. Nucleic Acids Res. 2017; 45:D712-D722. doi:10.1093/nar/gkw1128.

21. Jensen M, Cox AP, Chaudhry N, Ng M, Sule D, Duncan W, et al. The neurological disease ontology. J Biomed Semant. 2013; 4(1):42. doi:10.1186/2041-1480-4-42.

22. He Y, Sarntivijai S, Lin Y, Xiang Z, Guo A, Zhang S, et al. OAE: The ontology of adverse events. J Biomed Semant. 2014; 5(29). doi:10.1186/2041-1480-5-29.

23. Bandrowski A, Brinkman R, Brochhausen M, Brush MH, Bug B, Chibucos MC, et al. The Ontology for Biomedical Investigations. PLOS ONE. 2016; 11(4):e0154556. doi:10.1371/journal.pone.0154556.

24. OBI-NIAID-GSC-BRC-view. https://bioportal.bioontology.org/ontologies/NIAID-GSC-BRC. Accessed 27 Apr 2020.

25. Brochhausen M, Zheng J, Birtwell D, Williams H, Masci AM, Ellis HJ, et al. OBIB-a novel ontology for biobanking. J Biomed Semant. 2016; 7(23). doi: 10.1186/s13326-016-0068-y.

26. Ray P, Diehl AD. The ocular disease ontology. In: Dumontier M, Hoehndorf R, Baker CJO, editors. Proceedings of the $4^{\text {th }}$ International Conference on Biomedical Ontology (ICBO 2013). CEUR-WS.org; 2013. p. 119.

27. Schleyer TK, Ruttenberg A, Duncan W, Haendel M, Torniai C, Acharya A, et al. An ontology-based method for secondary use of electronic dental record data. AMLA Jt Summits Transl Sci Proc. 2013; p. 234 38.

28. Ontology of Host Pathogen Interactions. https://bioportal.bioontology.org/ontologies/OHPI. Accessed 27 Apr 2020.

29. Ontology of Laboratory Animal Medicine. https://bioportal.bioontology.org/ ontologies/OLAM. Accessed 27 Apr 2020.

30. Brochhausen M, Fransson MN, Kanaskar NV, Eriksson M, Merino-Martinez R, Hall RA, et al. Developing a semantically rich ontology for the biobank-administration domain. J Biomed Semant. 2013; 4(23). doi:10.1186/2041-1480-4-23.

31. Hicks A, Hanna J, Welch D, Brochhausen M, Hogan W. The ontology of medically related social entities: recent developments. J Biomed Semant. 2016; 7(47). doi:10.1186/s13326-016-0087-8.

32. Farinelli F, Almeida MB, Elkin PL, Smith B. OntONeo: The Obstetric and Neonatal Ontology. In: Jaiswal $\mathrm{P}$, Hoehndorf R, editors. Proceedings of the Joint International Conference on Biological Ontology and BioCreative (ICBO-BioCreative 2016). CEUR-WS.org; 2016. p. 35.

33. Ontology of Precision Medicine and Investigation https://bioportal.bioontology.org/ ontologies/OPMI. Accessed 27 Apr 2020.

34. Ethier JF, Barton A, Taseen R. An ontological analysis of drug prescriptions. Applied Ontology (2018) 13(4):273-294. doi:10.3233/ao-180202.

35. Planarian Phenotype Ontology. https://bioportal.bioontology.org/ontologies/PLANP. Accessed 27 Apr 2020.

36. He Y, Cowell LG, Diehl AD, Mobley H, Peters B, Ruttenberg A, et al. VO: Vaccine Ontology. In: Smith B, editor. Proceedings of the 1st International Conference on Biomedical Ontology (ICBO 2009). Buffalo: NCOR; 2009. p. 172. 
37. Goldfain A, Smith B, Arabandi S, Brochhausen M, Hogan W. Vital Sign Ontology. In: Proceedings of the Workshop on Bio-Ontologies, ISMB. 2011; p. 71-74.

38. Malaria Ontology. https:// github.com/VeuPathDB-ontology/IDOMAL. Accessed 27 Apr 2020.

39. Dengue Ontology. https://bioportal.bioontology.org/ontologies/IDODEN. Accessed 27 Apr 2020.

40. Sargeant D, Deverasetty S, Luo Y, Villahoz Baleta A, Zobrist S, Rathnayake V, et al. HIVToolbox, an integrated web application for investigating HIV. PLOS ONE. 2011; 6:e20122. doi: 10.1371/journal.pone.0020122.

41. Walls RL, Smith B, Elser J, Goldfain A, Stevenson DW, Jaiswal P. A plant disease extension of the infectious disease ontology. In: Cornet R, Stevens R, editors. Proceedings of the $3^{\text {rd }}$ International Conference on Biomedical Ontology. CEURS-WS.org; 2012. p. 1-5.

42. Ginsberg J, Mohebbi M, Patel R, Brammer L, Smolinski M, Brilliant L. Detecting Influenza Epidemics using Search Engine Query Data. Nature. 2009; 457(7232):2012-2014. doi:10.1038/nature07634.

43. Signorini A, Segre AM, Polgreen PM. The Use of Twitter to Track Levels of Disease Activity and Public Concern in the U.S. during the Influenza A H1N1 Pandemic. PLOS ONE. 2011; 6(5):e19467. doi:10.1371/journal.pone.0019467.

44. Generous N, Fairchild G, Deshpande A, Del Valle S, Priedhorsky R. Global disease monitoring and forecasting with Wikipedia. PLOS Comput Biol. 2014; 10(11):e1003892. doi: 10.1371/journal.pcbi.1003892.

45. Doan S, Ohno-Machado L, Collier N. Enhancing Twitter Data Analysis with Simple Semantic Filtering: Example in Tracking Influenza-Like Illness. In: Setti G, editor. HISB'12: Proceedings of the 2012 IEEE Health Care Informatics, Imaging and Systems Biology. Washington, DC: IEEE; 2012. p. 62-71. doi:10.1109/HISB.2012.21.

46. Magumba M, Nabende P, Mwebaze E. Ontology boosted deep learning for disease name extraction from Twitter messages. Journal of Big Data. 2018; 5(31). doi:10.1186/s40537-018 -0139-2.

47. Gordon CL, Pouch S, Cowell LG, Boland MR, Platt HL, Goldfain A, et al. Design and evaluation of a bacterial clinical infectious diseases ontology. AMLA Annu Symp Proc. 2013; p. 502- 511.

48. Lozano-Fuentes S, Bandyopadhyay A, Cowell LG, Goldfain A, Eisen L. Ontology for vector surveillance and management. J Med Entom. 2013; 50:1-14. doi:10.1603/me12169.

49. Matthews TC, Bristow FR, Griffiths EJ, Petkau A, Adam J, Dooley D, et al. The Integrated Rapid Infectious Disease Analysis (IRIDA) platform. bioRxiv. 2018; Available at: https://doi.org/10.1101/381830. Accessed 27 Apr 2020.

50. Xiang Z, Zheng W, He Y. BBP: Brucella genome annotation with literature mining and curation. BMC Bioinform. 2006; doi:10.1186/1471-2105-7-347.

51. Vaccine Investigation and Online Information Network. http://www.violinet.org/. Accessed 27 Apr 2020.

52. Cooper L, Meier A, Laporte MA, Elser J, Mungall CJ, Sinn BT, et al. The Planteome database: an integrated resource for reference ontologies, plant genomics and phenomics.

53. Eukaryotic Pathogen Genomics Database. https://eupathdb.org/eupathdb/. Accessed 27 Apr 2020.

54. VectorBase: Bioinformatics Resource for Invertebrate Vectors of Human Pathogens. http:/ /vectorbase.org. Accessed 27 Apr 2020.

55. https://eurekalert.org/pub_releases/2019-10/uop-nat101519.php. Accessed 27 Apr 2020.

56. Zheng J, Cade JS, Brunk B, Roos DS, Stoeckert CJ, Sullivan SA, et al. Malaria study data integration and information retrieval based on OBO Foundry ontologies. In: Jaiswal P, Hoehndorf R, editors. Proceedings of the Joint International Conference on Biological Ontology and BioCreative (ICBO-BioCreative 2016). CEUR-WS.org; 2016. p. 38.

57. Squires RB, Noronha J, Hunt V, García-Sastre A, Macken C, Baumgarth N, et al. Influenza Research Database: An integrated bioinformatics resource for influenza virus research. Influenza Other Respir Viruses. 2012; 6(6): 404-416. doi:10.1111/j.1750-2659.2011.00331.x.

58. https://bioportal.bioontology.org/projects/IRD

59. Virus Pathogen Resource. http://www.viprbrc.org.

60. Sayers S, Li L, Ong E, Deng S, Fu G, Lin Y, et al. Victors: a web-based knowledge base of virulence factors in human and animal pathogens. Nucleic Acid Res. 2019; 47:D693-D700. doi:10.1093/nar/ gky999. 
61. Zhang YF, Gou L, Zhou TS, Lin DN, Zheng J, Li Y, et al. An ontology-based approach to patient followup assessment for continuous and personalized chronic disease management. J Biomed Inform. 2017; 72:4559. doi: 10.1016/j.jbi.2017.06.021.

62. Abidi S. A knowledge-modeling approach to integrate multiple clinical practice guidelines to provide evidence-based clinical decision support for managing comorbid conditions. J Med Syst. 2017; 41(12):193. doi:10.1007/s10916-017-0841-1.

63. Lin Y, Staes CJ, Shields DE, Kandula V, Welch BM, Kawamoto K. Design, development, and initial evaluation of a terminology for clinical decision support and electronic clinical quality measurement. $A M L A$ Annu Symp Proc. 2015; p. 843-51.

64. Haendel MA, McMurry JA, Relevo R, Mungall CJ, Robinson PN, Chute CG. A Census of Disease Ontologies. Annu Rev of Biomed Data Sci. 2018; 1(1):305-331.

65. Thursky KA, Mahemoff M. User-centered design techniques for a computerized antibiotic decision support system in an intensive care unit. Int J Med Inform. 2007; 76:760-8.

66. Paterson DL. The role of antimicrobial management programs in optimizing antibiotic prescribing within hospitals. Clin Infect Dis. 2006; 42 Suppl 2: S90-5. doi:10.1086/499407.

68. Shen Y, Yuan K, Chen D, Colloc J, Yang M, Li Y, et al. An ontology-driven clinical decision support system (IDDAP) for infectious disease diagnosis and antibiotic prescription. Artif Intell Med. 2018; 86:2032.

69. Eisen L, Coleman M, Lozano-Fuentes S, McEachen N, Orlans M, Coleman M. Multi-disease data management system platform for vector-borne diseases. PLOS Negl Trop Dis. 2011; 5:e1016. doi:10.1371/journal.pntd.0001016.

70. Lozano-Fuentes S, Barker CM, Coleman M, Park BB, Reisen WK, Eisen L. Emerging information technologies to provide improved decision support for surveillance, prevention, and control of vectorborne diseases. In: Jao C, editor. Efficient Decision Support Systems: Practice and Challenges in Biomedical Related Domain. Rijeka, Croatia: InTech-Open Access Publisher; 2011. p. 89-114.

71. Lozano-Fuentes S, Wedyan F, Hernandez-Garcia E, Devadatta S, Ghosh S, Bieman JM, et al. Cell phonebased system (Chaak) for surveillance of immatures of dengue virus mosquito vectors. J Med Entomol. 2013; 50(4):879-89. doi:10.1603/me13008.

\section{Additional File 2 \\ The Infectious Disease Ontology Extensions: Some Issues}

\section{Shane Babcock and John Beverley}

As we move forward in our efforts to refine the IDO suite, many of the IDO extensions will require reengineering. Here we detail some of the issues that need to be addressed within specific IDO extensions.

\section{The Coronavirus Infectious Disease Ontology (CIDO)}

Alignment with IDO Core will require much curation. For example, in CIDO:

- Created terms do not have textual definitions

- There are two distinct hierarchies, one for entity and one NCBITaxon:taxonomic rank

Within the entity subclass BFO:continuant.

- ChEBI:role is as a sibling class of BFO:role, and distinct definitions are provided

- OBI:host role is a sibling class of IDO:host role

- OGMS:disease is a sibling class of NDF-RT:Diseases, Manifestations, or Physiological States 
- Related, the imported class IDO:infectious disorder has no child terms, despite coronavirus infections being in purview of CIDO

- Moreover, CIDO has not introduced relevant subclasses of IDO: host such as IDO:pathogen host or IDO:intermediate host, presumably needed to track the spread of coronaviruses

- IDO:pathogen has only the subclass IDO:infectious agent, though further specification is expected in CIDO given its focus on viruses

- ChEBI:antiviral agent is imported as a BFO:role but there is no corresponding material entity bearer; similarly for ChEBI:antiviral drug; CIDO does import VO:viral vaccine but vaccines are not antivirals

- ChEBI:molecular entity is defined as a direct subclass of both BFO:material entity and BFO:object

- CIDO inherits from UBERON the class anatomical entity as a subclass of BFO:material entity and yet is defined as either a material or immaterial entity; this is reflected in CIDO also importing under BFO:material entity the UBERON class material anatomical entity. BFO:material entity is disjoint from BFO:immaterial entity, though material entities may have immaterial parts

Turning to the entity subclass BFO:occurrent:

- CIDO only imports BFO:process as a child class of BFO:occurrent, and so lacks the resources to specify temporal intervals and points of time, which thereby undermines CIDO's ability to represent the spread of coronaviruses over time; this suggests CIDO is unable to represent antivirals specifically targeting process parts of coronavirus reproduction, as discussed in the main document

- In particular, CIDO cannot obviously at this point represent the coronavirus reproduction cycle

- Moreover, OGMS: disease course has only the subclass IDO:infectious disease course, lacking further expected specification for coronaviruses

- CIDO does, however, create terms for coronavirus, e.g. CIDO:coronavirus infectious disease process and sibling subclasses such as CIDO: $S A R S-C o V$ disease process and CIDO:Human coronavirus 229E DP - all processes - some of which are related to the other coronavirus terms that have been imported from, say DOID, and other ontologies, but not all

Turning lastly to object properties:

- CIDO's relation CIDO:is host of is not defined

Concerning this last point, IDO Core provides resources that might be useful for the construction of the needed definition. CIDO imports IDO:host and IDO:infectious agent, and could also import IDO:infectious structure, presumably the relata of this relation. Recall that IDO Core defines a host as an organism having some material entity as part or as part of its extended organism. As a first pass, one might define:

- $\quad$ is host of $=_{\text {def }}$ host $\mathrm{x}$ is part of extended organism $\mathrm{z}$ and $\mathrm{z}$ has proper part material entity $\mathrm{y}$ and $\mathrm{x} \neq \mathrm{y}$

CIDO might then sharpen this to a sub-relation holding between a host and a virus that is an infectious structure, which makes sense given the domain of interest: 
- $\quad$ is host of virus $=_{\text {def }}$ host $\mathrm{x}$ is host of virus $\mathrm{y}$ and $\mathrm{y}$ is an infectious structure

That said, this relation is of limited use, since it would be false to assert anything stronger than some host is the host of some virus infectious agent. In particular, it would be false to assert that all hosts are hosts of some virus infectious agent. We might define instead for CIDO the following variation, relying on the BFO:part of relation:

- virus has host $=_{\text {def }} \mathrm{x}$ is a virus and $\mathrm{x}$ is an infectious structure and has established an infection in proper part host $\mathrm{y}$ of extended organism $\mathrm{z}$ and $\mathrm{x} \neq \mathrm{y}$

Which allows one to make the true claim that all virus infectious structures that have established an infection have some host.

CIDO developers intend to align with IDO and relevant, related, ontologies, e.g. OGMS, BFO. Presently, CIDO and IDO developers are working to ensure alignment.

\section{Brucellosis Ontology (IDOBRU)}

Though IDOBRU is largely in alignment with IDO Core and other relevant ontologies, there are some aspects of the ontology needing to be addressed. We have begun to address some of these issues. For example:

- IDOBRU previously contained the term brucellosis disposition, redundantly defined as "A brucellosis disposition that is the disposition to be transmitted from an infected non-human host to a human host", while its parent IDO:zoonotic disposition is defined as "the disposition to be transmitted from an infected non-human host to a human host". IDOBRU:brucellosis disposition should not be a child of IDO: zoonotic disposition, given that Brucella can be transmitted from humans to humans in addition to being transmitted zoonotically. To fix this issue, we replaced brucellosis disposition with the new term Brucella infectious disposition, which we define as follows:

Brucella infectious disposition $=\operatorname{def}$ An infectious disposition that is the disposition of Brucella to be transmitted to a host and establish a Brucella infectious disorder.

Since a Brucella infectious disorder is the material basis of the brucellosis disease, this definition captures the fact that Brucella is a cause of brucellosis. For zoonotic transmission, we may also define a new term, brucella zoonotic disposition, along the following lines:

Brucella zoonotic disposition $=\operatorname{def}$ An infectious disposition that is the disposition of Brucella to be transmitted from an infected non-human host to a human host.

Several textual definitions are circular:

- IDOBRU:brucellosis pathogen role is a subclass of IDO:pathogen role, but the former was previously defined circularly as "a pathogen role of being a brucellosis pathogen". We have revised this definition by refinement of the differentia used to define IDO:pathogen role, replacing the term pathogenic disposition with Brucella infectious disposition, as follows: 
brucellosis pathogen role $=_{\text {def }}$ A pathogen role borne by Brucella bacterium when contained in a host in which its Brucella infectious disposition can be realized.

Other IDO extensions could provide similar definitions for pathogen roles corresponding to Staphylococcus aureus, the influenza viruses, malaria parasites, and so on.

- IDOBRU:veterinarian role is also circularly defined as "a role of being a veterinarian", and similar remarks apply to IDOBRU:animal dealer role, IDOBRU:bioterrorism agent role, IDOBRU:butcher role, and several others

- IDOBRU:drinking function is similarly circularly defined as "the drinking function of realizing a drinking disposition"

Some classes have lone children, such as:

- IDOBRU:process of establishing Brucella infection in host is the sole child of IDO:process of establishing an infection

Of note, IDO Core developers have begun working with IDOBRU developers to align the epidemiology related content of the two ontologies. As noted in the main text, IDO Core contains qualities of disease affected populations, such as infectious disease incidence rate, infectious disease mortality rate, and infectious disease endemicity. We have recently added terms for the corresponding sites at which these qualities are instantiated. For example: infectious disease endemic site, infectious disease free site, and infectious disease non-endemic site. Adding these classes allows them to serve as parent classes for the brucellosis specific classes brucellosis endemic site, brucellosis free site, and brucellosis non-endemic site, currently used in IDOBRU.

III. Influenza Ontology (FLU)

As there are no previous publications in which FLU has been described we discuss its content in some detail, as well as our recent efforts to achieve alignment with IDO Core. In the most recent published version of $\mathrm{FLU}^{4}$ (dated Aug 20, 2015), classes are currently divided between two separate is_a hierarchies. One of these is built upon the outdated BFO version 1.1. The other is built upon BFO version 2.0. For the purposes of our exposition, we refer to these hierarchies as FLU1.1 and FLU2.0 respectively.

Below is a summary of some of the FLU terms appearing within FLU1.1, organized according to the BFO version 1.1 classes under which they fall:

- FLU terms descending from material entity include: terms related to influenza surveillance such as bird identification band; terms for influenza proteins such as influenza hemagglutinin and influenza neuraminidase; terms relating to influenza prevention and treatment such as organism treated with antibiotics, organism treated with antiviral drug, and vaccinated organism

- Subclasses of OBI:organism such as Influenza virus $A$, Influenza virus $B$, and Influenza virus $C$

- A variety of terms for influenza host organisms descending from NCBITaxon:Eukaryota.

${ }^{4}$ https://bioportal.bioontology.org/ontologies/FLU/ 
- FLU terms descending from role include a variety of terms relating to influenza surveillance and sequencing such as extractor role, sequence annotator role, specimen collector role (which appear as direct children of OBI:investigation agent role)

- Descending from quality is the FLU term life stage, which has several children including fledgling, hatch year, nestling and yearling (for the representation of life stages of avian influenza hosts)

In future iterations of FLU, FLU1.1 terms and children will be reassigned to the corresponding $\mathrm{BFO}$ version 2.0 classes. Notable challenges include:

- FLU1.1's planned process hierarchy is identical to that of FLU2.0, except in the former OBI:planned process descends from the outdated BFO1.1 term processual entity

- FLU1.1 OBI:planned process has sibling classes that do not appear in FLU2.0, such as FLU:enrichment process, OGMS: health care process, and OGMS: treatment.

- In both planned process hierarchies of FLU2.0 and FLU1.1, the OBI:collecting specimen from organism and OBI:environmental material collection are children of FLU:specimen creation, defined as "a planned process with the objective of obtaining specimen." However, in FLU1.1, but not FLU2.0, the same OBI classes also appear as children of the functionally equivalent FLU:specimen collection, which is defined as "the process of collecting a specimen, either from an organism or from an environment"

This is list is incomplete and is just a snapshot of the terms that require reassignment.

Below is a summary of some of the FLU terms appearing within FLU2.0. All of those that were newly created for FLU descend either from IAO:information content entity (under BFO:generically dependent continuant) or from OBI:planned process (under BFO:process):

- Under the IAO:information content entity subclass IAO:data item there are a variety of FLU terms representing data items pertaining to influenza surveillance and influenza virus sequences. Subclasses of IAO:data item include data about a collection location, data about a genbank entry, nucleic acid sequence, and influenza virus antigenic data

- IAO:scalar measurement datum has as subclasses FLU terms such as hemagglutinin titer, sequence coverage, initial oxygen saturation and virus pathogenicity after passage

- IAO:plan specification includes a variety of FLU terms pertaining to influenza surveillance and virus sequencing such as virus isolation protocol, sequencing protocol, and specimen collection protocol

- FLU imports the IAO terms material information bearer, photographic print, information carrier, and author role (the first two as children of BFO:material entity, the third as a child of BFO:quality and the fourth as a child of BFO:role)

- OBI:planned process subclass OBI:assay has as children FLU terms including hemagglutinin typing and neuraminidase typing. Both of have the child OBI:viral hemagglutination inbibition assay

- OBI:assay subclass OBI:sequencing assay has the FLU term genomic sequencing as a child

- Direct or indirect FLU subclasses of OBI:material component separation include virus amplification, virus passage and virus isolation

- FLU:virus isolation is a child of OBI:extraction (itself a child of OBI:material component separation). 
While obviously relevant to the influenza domain, many of the data related FLU terms listed above apply to viral diseases more generally. Likewise, the FLU processual entities genomic sequencing, virus amplification, virus passage, virus isolation, and antiviral treatment are not specific to the influenza domain.

FLU also has some issues with regard to what is defined and what is expected to be defined but not. For example:

- FLU:organism treated with antiviral drug is defined as "an organism that has been treated with an antiviral drug." But there is no defined relation treated by, and the use of "drug" is ambiguous, i.e. between meaning a material entity, or meaning a role of some material entity, the latter used by ChEBI from which FLU imports several terms

- FLU:antiviral drug has one child, FLU: neuraminidase inbibitor drug defined as "A drug targeted at the influenza virus, which works by blocking the function of the viral neuraminidase protein, thus preventing the virus from reproducing by budding from the host cell." IDO Core recommends explicating phenomena used in the definition in part as involving dispositions. Yet, antiviral disposition is not currently present within FLU

- IDO:antibacterial is defined in terms of the class IDO: antibacterial disposition, but this class has not been included in FLU

- More generally, FLU includes only one disposition: FLU: hemagglutination disposition defined as the disposition to initiate hemagglutination processes, yet introduces a class FLU:organism treated with antiviral drug simply defined as an organism that has been treated by an antiviral drug; IDO Core terms like protective resistance, to characterize flu strain resistance to antiviral drug treatments would surely be useful here

We are currently developing an updated version of FLU, which can be found here:

https://github.comPhiBabs935/IDO-FLU. As part of this project, we have reassigned the following FLU terms to VIDO: virus isolation protocol, virus amplification, amplified virus preparation, virus passage, virus passage date, virus pathogenicity after passage, virus isolation, antiviral treatment, and antiviral treatment date. After searching ONTOBEE, we found that a variety of OBO Foundry and Library ontologies contained existing terms that were suitable to replace FLU terms that were out of scope. These ontologies, to name a few, include the Ontology of Biomedical Investigations (OBI ${ }^{6}$, the VEuPathDB Ontology (EUPATH), the Genomic Epidemiology Ontology (GenEpio), and the Vaccine Ontology (VO). To give just a few examples of the relevant term replacements: FLU:specimen collection, FLU:sequencing protocol, FLU:sequence coverage, and FLU:vaccinated organism have been replaced, respectively, with OBI:specimen collection process, EUPATH:sequencing protocol, GenEpio:read coverage, and VO:vaccinated organism. In our updated version of FLU, all obsoleted FLU terms can be found under Obsolete Class with annotations pointing to the higher-level ontology term with which they have been replaced.

Where suitable replacements could not be found, we have made requests to have various FLU terms ported out to more appropriate upper-level OBO Foundry ontologies. First instance, we have suggested exporting a number of terms to both OBI and GenEpio. Thus, see the following

\footnotetext{
${ }^{5}$ Note, as the term FLU:antiviral drug has applicability to viral infections in general, in our developmental version of FLU we have replaced this term with ChEBI:antiviral drug.

${ }^{6}$ The majority of existing replacement terms were taken from OBI. This is not a surprise, as a variety of persons associated with the development of OBI helped develop FLU. It appears that many of the terms that were created for FLU very early on - prior to OBI's creation - were succeeded by more or less functionally equivalent OBI terms.
} 
issue trackers: https://github.com/obi-ontology/obi/issues/1286; and: https://github.com/ GenEpiO/genepio/issues/22. Upon request, the term $\mathrm{RN} A$ extraction protocol was added to the Experimental Factor Ontology (EFO), and then imported to replace FLU:RNA extraction protocol (see: https://github.com/EBISPOT/efo/issues/940). Related, we have imported a number of existing EFO terms to replace out of scope FLU terms (e.g., FLU:extraction protocol has been replaced by an equivalently labeled term from EFO.)

We have also created many new influenza related terms for FLU and have also imported many new terms to cover facets of the domain not covered in previous versions of the ontology. In doing so we have reengineered FLU as a direct extension of VIDO. Many of the new terms that were created for FLU mirrored the creation of comparable terms for IDO-COVID-19.

\section{The HIV Ontology (HIV)}

HIV imports neither BFO nor IDO Core; HIV terms are not organized into a hierarchy, and the majority are direct children of OWL:thing, i.e. the ontology is primarily a flat list of terms having few child classes. If it is to be considered an extension of IDO Core, the HIV ontology will need significant updating and re-organization of its content. Indeed, it is difficult to make suggestions for alignment with IDO Core, without the presence of any hierarchy characteristic of ontologies.

While the ontology only focuses on HIV virus biology and genetics, with a few terms pertaining to antiviral drugs and resistance. There is no coverage of terms pertaining to transmission or pathogen hosts.

\section{The Malaria Ontology (IDOMAL) and The Dengue Ontology (IDODEN)}

As noted in the main text, IDOMAL has recently been obsoleted. It will eventually be replaced with a new IDO extension for malaria. That said, we believe that careful consideration of IDOMAL's errors will be instructive for when we begin to develop its future replacement. In the following, for the sake of brevity we will refer to this future replacement as MAL 2.0. While IDOMAL terms will not be reused, there is still much useful information represented in the ontology that can be used as a loose template for constructing MAL 2.0.

As IDOMAL and IDODEN were built according to the same architecture, they share the same alignments and discrepancies with IDO Core. Thus, we consider the two ontologies together. Each is a well-organized hierarchy, but that hierarchy is built on BFO1.1 rather than BFO2.0. Moreover, though many key terms are imported from IDO Core, neither inherit IDO Core's hierarchical structure. Alignment with IDO Core clearly requires importing relevant terms consistent with IDO Core's hierarchy.

One persistent problem worth discussion is the conflation of entity types. In some cases, qualities and realizable entities are conflated with occurrent entities, and in other cases dispositions are conflated with qualities. For example, in both ontologies:

- symptom is a subclass of condition, itself a child of BFO:realizable entity. But several subclasses of symptom are processual entities, conflating processes and realizable entities

- RO:bappens_during, which is a relation between processes, is often asserted as holding between continuants, e.g. IDOMAL:circulatory collapse is a subclass of symptom and happens_during some 
clinical manifestation of falciparum malaria, but the latter is a subclass of quality of malaria;

IDODEN:ascites is a subclass of symptom and happens_during some clinical manifestation of dengue, but the latter is a subclass of quality of dengue fever

- BFO:continuant entity is conflated with BFO:occurrent entity, e.g. IDOMAL:clinical manifestation of malaria is a subclass of part_of some progression of malaria and IDODEN:clinical manifestation of dengue is asserted as a progression of dengue fever, so in each case a quality is asserted as a part of some process, but BFO:continuant entity can be a part of some BFO:occurrent entity

- contagiousness is defined as an ability to spread from one person or species to another, and yet IDODEN follows IDO Core in classifying contagiousness as a disposition, while IDOMAL classifies it as a quality of malaria

- zoonotic, defined as "a disease that can be transmitted from animals to people", is classified in IDOMAL as a quality of malaria, and in IDODEN as a quality of dengue fever

The last two examples highlight a major issue arising from the reliance on placeholder classes like quality of malaria and quality dengue fever, defined, respectively, as "A quality that refers to malaria" and as "A quality that refers to dengue fever". Such placeholder classes often result in the inheritance of properties that do not hold for a type. Thus, while contagiousness is a subtype of quality of malaria in IDOMAL, not all instances of contagiousness refer to malaria. The same point applies to the classification of zoonotic as a quality of malaria in IDOMAL and its classification as quality of dengue fever in IDODEN. Similarly, in both IDOMAL and IDODEN, immunity is classified as a quality of host, which is defined as "A quality which refers to the host". But not all instances of immunity refer to a host, as an organism can have an immunity without being a host. Or consider the class quality of vector, defined as "A quality which refers to vectors." In both IDOMAL and IDODEN there are many classes, including flight capacity, male fecundity, and zoophily, which are classified as subtypes of quality of vector even though instances of these types can be, and are, present in insects that do not happen to be vectors.

IDOMAL does contain many terms, for which newly created variants should be added to MAL 2.0. But the introduction of such terms will require the correction of some issues. For example:

- IDOMAL:antiparasitic chemical compound is defined as "A chemical compound bearing an antiparasitic disposition" and includes comprehensive subclasses, but since IDOMAL does not import IDO, this is not a subclass of IDO:antiparasitic, but rather of ChEBI:chemical compound. This issue of course can easily be fixed by introducing IDO:antiparasitic into MAL 2.0 and populating this class with new replacements for IDOMAL:antiparasitic chemical compound and its subclasses. Likewise, for similar cases.

- IDOMAL:malaria vector is a subclass of host role defined as "An anapheline mosquito transmitting malaria". Strictly speaking, a malaria vector is a transporter of the pathogens of malaria. For MAL 2.0, malaria pathogen-specific child terms are needed for infectious agent vector role.

The same can be said for many classes descending from IDOMAL's problematic placeholder classes. In MAL 2.0 replacement terms for these classes will be created and be reassigned, where possible, to hierarchies descending from appropriate upper-level terms from IDO Core. Where this is not possible, the IDO Core developers will discuss the possibility of adding new classes to IDO Core. 
Recently, IDOMAL was inherited by the Stoeckert team at the University of Pennsylvania, which is hosting the ontology for legacy purposes here: https://github.com/VeuPathDBontology/IDOMAL. As part of a recently funded initiative to revamp and integrate the Eukaryotic Pathogen Genomics Database (https://eupathdb.org/eupathdb/) and the Bioinformatics Resource for Invertebrate Vectors of Human Pathogens (VectorBase: http://vectorbase.org), the Stoeckert team has replaced many terms with IDOMAL prefixes that were previously used to annotate data in VectorBase - which are not really infectious disease terms per se, and so should not have IDOMAL prefixes - with terms drawn from other OBO Foundry ontologies. Specifically:

IDOMAL:0000129 (Plasmodium falciparum), IDOMAL:0000131 (Plasmodium ovale), IDOMAL:0000132 (Plasmodium vivax), IDOMAL:0000133 (Plasmodium malariae), IDOMAL:0000222 (enzyme-linked immunosorbent assay), IDOMAL:0000443 (gravid), IDOMAL:0000552 (Giemsa staining), IDOMAL:0000653 (larva), IDOMAL:0000654 (pupa), IDOMAL:0000655 (adult), IDOMAL:0000658 (third instar larva), IDOMAL:0000659 (fourth instar larva), IDOMAL:0001254 (population).

They are now using NCBITaxon for the species, OBI for the assay related terms and for population, and UBERON for the life stages.

Of note, to represent the varying degrees to which diseases can be endemic within different populations, we have introduced to IDO Core the following epidemiology terms which were originally created for IDOMAL: boloendemicity, hypoendemicity, and mesoendemicity.

\section{The Schistosomiasis Ontology (IDOSCHISTO)}

IDOSCHISTO will require significant rebuilding if it is to be considered a bona fide extension of IDO Core. IDO CORE is imported in full, and certain key classes descend from IDO Core classes (e.g. Schistosoma is_a IDO:infectious agent, Schistosomiasis is_a IDO:infectious disease). Still, most IDOSCHISTO terms lack textual definitions, and many other classes fall outside the BFO entity hierarchy entirely. Rather, they appear as subclasses of classes that are themselves direct children of OWL:thing. For example:

- sample_for_direct_diagnosis

- snail_survival_condition

- schistosoma_survival_condition

- schistosomiasis_pathological_process

While the ontology's developers acknowledge the importance of reusing terms from established OBO Foundry ontologies, in various cases IDOSCHISTO fails to adhere to the principle of orthogonality. For example:

- IDOSCHISTO:drug and IDOSCHISTO:vaccine were newly created instead of reusing CHEBI:drug and VO:vaccine

Dr. Camara has recently informed us (personal communication) that he and his team hope to soon begin a new project studying schistosomiasis and fasciolosis in Senegal. In coordination with this 
project they have plans to revise and update IDOSCHISTO. We will be in communication with their team to help ensure consistency and IDO Core conformity.

VII. The Meningitis Ontology (IDOMEN)

IDOMEN, in its current iteration, suffers from several issues, and is far from alignment with BFO, OGMS, and IDO Core despite importing each in full. Of importance:

- IDOMEN is exceedingly difficult to navigate, due to the redundant assertion of classes

- IDOMEN's continuant classes all appear as direct children of BFO:continuant the same can be said for its occurrent hierarchy)

- IDOMEN sometimes fails to adhere to the principle of orthogonality, for example IDOMEN adds the new terms patient, patient role, and vaccination to serve, respectively, as the parents of the IDOMEN terms meningitis patient, meningitis patient, and vaccination campaign rather than import OAE:patient, OBI:patient role, and VO:vaccination. Similarly, to represent meningitis epidemic risk factors, IDOMEN adds a number of new terms for environmental factors, including terms for climatic factors. IDOMEN should consider reusing terms from ENVO

- Many IDOMEN terms lack textual definitions

- IDO:infectious disposition has the sole child IDOMEN:meningitis disposition

\section{The importation of disease terms from the Human Disease Ontology (DOID)}

While some IDO extensions import disease terms from DOID we do not recommend this in all cases moving forward. DOID purports to follow OGMS. While many DOID disease terms are defined in terms of underlying disorders, this is not so in all cases. In general, DOID disease terms often contain more information than is appropriate. Consider for instance DOID: influenza:

influenza $=$ def. A viral infectious disease that results in infection, located in respiratory tract, has_material_basis_in Influenzavirus A, has_material_basis_in Influenzavirus B, or has_material_basis_in Influenzavirus C, which are transmitted by droplet spread of oronasal secretions during coughing, sneezing, or talking from an infected person. It is a highly contagious disease that affects birds and mammals and has symptom chills, has symptom fever, has symptom sore throat, has symptom runny nose, has symptom muscle pains, has symptom severe headache, has symptom cough, and has symptom weakness.

A good definition captures just those essential features of the class, including its genus, as well as the differentia that distinguishes it from other subtypes of that genus. Most of the features included in the above definition are not differentia. Location in the respiratory tract, transmissibility through droplet spread, and many of the mentioned symptoms, are features common to a variety of viral infectious diseases. Such information would be better relegated to the term's associated OWL axioms, or an editor's comment. Notice the definition also gets things backwards by saying that influenza results in an infection. Rather, the infection is the basis of the disease. A better, OGMSbased template is provided by IDOSA: Staphylococcus aureus infectious disease:

Staphylococcus aureus infectious disease $=$ def. Infectious disease that has a staphylococcus aureus infectious disorder as its material basis. 
Accordingly, influen₹a ought to be defined as follows:

influenza $=$ def. Viral infectious disease that has as its material basis either an Influenzavirus A infectious disorder, an Influenzavirus B infectious disorder, or an Influenzavirus C infectious disorder.

\section{Additional File 3 \\ Case Study: IDOSA and methicillin resistant Staphylococcus aureus}

IDO:protective resistance is used to model the resistance of certain bacteria to antibiotic drugs. For this purpose, the following subtypes of protective resistance are asserted in IDO Core:

drug resistance $=$ def. Protective resistance that mitigates the damaging effects of a drug. antibiotic resistance $=$ def. Drug resistance that mitigates the damaging effects of an antibiotic.

Beta-lactam antibiotics such as methicillin are the most widely used antibiotics, and most work by preventing bacterial cell wall construction. They act by binding to and inhibiting the penicillinbinding-proteins (PBPs) within bacteria that facilitate the synthesis of peptidoglycan molecules, thus compromising the structural integrity of the cell wall. In response to the widespread use of betalactam antibiotics, some bacteria have rapidly evolved novel-structured PBPs which lack an affinity for these antibiotics, thus rendering them less effective. In IDO we aim to provide an ontological representation of resistance that reveals the active mechanisms that produce resistance-in the case of resistant bacteria, the active dispositions inhering in novel-structured PBPs that inhibit antibiotics from manifesting their damaging effects [1]. 
Consider the case of methicillin resistant Staphylococcus aureus (MRSa). MRSa's resistance to methicillin is conferred by PBP2a, a PBP that lacks affinity for methicillin and is the product of the gene mec $A$. The need to provide a coherent and consistent understanding of the mechanisms underlying MRSa antibiotic resistance is one impetus for the development of the Staphylococcus aureus Infectious Disease Ontology (IDOSA), an extension of IDO covering entities specific to Staph aureus $(\mathrm{Sa})$ infectious diseases $[1,2]$.

IDOSA's main hierarchy is built on BFO, and imports IDO Core in full. IDOSA provides terms covering all entities relevant to antibacterial resistance in Sa, including terms for Sa proteins (from the Protein Ontology [3]), terms for genes and gene products, (from the Sequence Ontology [4]) terms for biological processes (from the GO Biological Process Ontology), terms for anatomical sites of infection (from the UBERON anatomy ontology [5]), and terms for antibiotics (from Chemical Entities of Biological Interest (ChEBI) [6]).

IDOSA imports the term staphylococcus aureus from NCBITaxon, while adding the following subclasses:

methicillin-resistant Staphylococcus aureus =def. Organism of type Staphylococcus aureus that has resistance to beta-lactam antibiotics.

methicillin-susceptible Staphylococcus aureus =def. Organism of type Staphylococcus aureus that is susceptible to beta-lactam antibiotics.

Both are defined in terms of IDOSA resistance to beta-lactam antibiotic, which itself is a subclass of IDO:antibiotic resistance and defined as follows:

resistance to beta-lactam antibiotic $=$ def. Antibiotic resistance that mitigates the damaging effects of a beta-lactam antibiotic.

With these terms and definitions, we can characterize both Methicillin-susceptible Staphylococcus aureus's (MSSa) susceptibility, and MRSa's resistance, to beta-lactam antibiotics in terms of protective resistance and blocking dispositions [1]).

MSSa is susceptible to the damaging effects of methicillin because it lacks protective resistance to that drug. Characterized positively, MSSa's PBPs have the disposition to undergo a methicillin PBP binding process that negatively_ regulates the synthesis of peptidoglycan, thereby interfering with the formation of a stable cell wall. Affinity for methicillin thus acts as a blocking disposition for the PBPs' disposition to synthesize peptidoglycan.

In the case of MRSa, in contrast, the disposition of its PBP2a parts to synthesize peptidoglycan, and thereby participate in the construction of a stable cell wall (which negatively_regulates methicillin binding), cannot be blocked. Thus, MRSa's protective antibiotic resistance to methicillin can be seen as an active response in which PBP2a manifests a disposition to mitigate the damaging effects of methicillin. [2] shows how the formal representation of these relations can be used in association with instance data to draw inferences that may facilitate automated drug discovery and guide treatment decisions in specific types of cases.

The IDO Core account of protective resistance can be applied also to other cases, such as the resistance against HIV-1 conferred by CCR $5-\Delta 32$, and the resistance against malaria conferred by the sickle cell trait [1]. CCR $5-\Delta 32$ is a deletion mutation of the CCR5 gene resulting in cells which lack a functioning CCR 5 receptor on their surfaces. In this case, the disposition of individuals with the CCR5- $\triangle 32$ mutation to develop cells that lack CCR 5 on their surface acts as a blocking disposition for the disposition of HIV-1 to bind to a CCR5 molecule. Plasmodium falciparum, one of the infectious agents that causes malaria, has a disposition to spread through the host by replicating within the host's red blood cells, lysing from those cells, and then entering again into further red 
blood cells - a process that is reduced in dense, dehydrated red blood cells. In individuals with the sickle cell hemoglobin gene, red blood cells have a disposition to become dehydrated and thus increase in density. This disposition acts as a blocking disposition for the disposition of plasmodium to spread through red blood cells, a process requiring hydrated red blood cells.

\section{References}

1. Goldfain A, Smith B, Cowell LG. Towards an ontological representation of resistance: the case of MRSA. J Biomed Inform. 2011; 44:35-41. doi:10.1016/j.jbi.2010.02.008.

2. Goldfain A, Smith B, Cowell LG. Constructing a lattice of infectious disease ontologies from a staphylococcus aureus isolate repository. In: Cornet R, Stevens R, editors. Proceedings of the $3^{\text {rd }}$ International Conference on Biomedical Ontology (ICBO 2012). CEURS-WS.org; 2012. P. 1-5.

3. Natale DA, Arighi CN, Blake JA, Bona J, Chen C, Chen S, et al. Protein Ontology (PRO): enhancing and scaling up the representation of protein entities. Nucleic Acids Res. 2017; 45: D339-D346. Doi: $10.1093 / \mathrm{nar} / \mathrm{gkw} 1075$.

4. Eilbeck K, Lewis SE, Mungall CJ, Yandell M, Stein L, Durbin R, et al. The Sequence Ontology: a tool for the unification of genome annotations. Genome Biol. 2005; 6(5):R44. doi: 10.1186/gb-2005-6-5-r44.

5. Haendel MA, Balhoff JP, Bastian FB, et al. Unification of multi-species vertebrate anatomy ontologies for comparative biology in Uberon. J Biomed Semant. 2014; doi:10.1186/2041-1480-5-21.

6. Degtyarenko K, Matos P, Ennis M, et al. ChEBI: a database and ontology for chemical entities of biological interest. Nucleic Acids Res. 2008; 36:D344-D350. doi:10.1093/nar/ gkm791. 